\title{
High-energy scaling of Compton scattering light sources
}

\author{
F. V. Hartemann, W. J. Brown, D. J. Gibson, S. G. Anderson, A. M. Tremaine, P. T. Springer, A. J. Wootton, \\ E. P. Hartouni, and C. P. J. Barty \\ Lawrence Livermore National Laboratory, Livermore, California 94550, USA
}

(Received 11 November 2004; published 31 October 2005)

\begin{abstract}
No monochromatic $\left(\Delta \omega_{x} / \omega_{x}<1 \%\right)$, high peak brightness $\left[>10^{20}\right.$ photons $/\left(\mathrm{mm}^{2} \times \operatorname{mrad}^{2} \times \mathrm{s} \times\right.$ $0.1 \%$ bandwidth)], tunable light sources currently exist above $100 \mathrm{keV}$. Important applications that would benefit from such new hard x-ray and $\gamma$-ray sources include the following: nuclear resonance fluorescence spectroscopy and isotopic imaging, time-resolved positron annihilation spectroscopy, and $\mathrm{MeV}$ flash radiography. In this paper, the peak brightness of Compton scattering light sources is derived for head-on collisions and found to scale quadratically with the normalized energy, $\gamma$; inversely with the electron beam duration, $\Delta \tau$, and the square of its normalized emittance, $\varepsilon$; and linearly with the bunch charge, $e N_{e}$, and the number of photons in the laser pulse, $N_{\gamma}: \hat{B}_{x} \propto \gamma^{2} N_{e} N_{\gamma} / \varepsilon^{2} \Delta \tau$. This $\gamma^{2}$ scaling shows that for low normalized emittance electron beams $(1 \mathrm{nC}, 1 \mathrm{~mm} \cdot \mathrm{mrad},<1 \mathrm{ps}$, $>100 \mathrm{MeV})$, and tabletop laser systems (1-10 J, $5 \mathrm{ps})$ the x-ray peak brightness can exceed $10^{23}$ photons $/\left(\mathrm{mm}^{2} \times \mathrm{mrad}^{2} \times \mathrm{s} \times 0.1 \%\right.$ bandwidth $)$ near $\hbar \omega_{x}=1 \mathrm{MeV}$; this is confirmed by threedimensional codes that have been benchmarked against Compton scattering experiments performed at Lawrence Livermore National Laboratory. The interaction geometry under consideration is head-on collisions, where the x-ray flash duration is shown to be equal to that of the electron bunch, and which produce the highest peak brightness for compressed electron beams. Important nonlinear effects, including spectral broadening, are also taken into account in our analysis; they show that there is an optimum laser pulse duration in this geometry, of the order of a few picoseconds, in sharp contrast with the initial approach to laser-driven Compton scattering sources where femtosecond laser systems were thought to be mandatory. The analytical expression for the peak on-axis brightness derived here is a powerful tool to efficiently explore the 12-dimensional parameter space corresponding to the phase spaces of both the electron and incident laser beams and to determine optimum conditions for producing highbrightness $\mathrm{x}$ rays.
\end{abstract}

DOI: 10.1103/PhysRevSTAB.8.100702

PACS numbers: 41.60.Ap, 41.60.Cr, 52.59.Px, 07.85.Fv

\section{INTRODUCTION}

No monochromatic $\left(\Delta \omega_{x} / \omega_{x}<1 \%\right)$, high peak brightness $\left[>10^{20}\right.$ photons $/\left(\mathrm{mm}^{2} \times \mathrm{mrad}^{2} \times \mathrm{s} \times 0.1 \%\right.$ bandwidth $\left.)\right]$, tunable light sources currently exist above $100 \mathrm{keV}$; yet a wide array of important applications would greatly benefit from such novel hard x-ray and gamma-ray sources, including nuclear fluorescence spectroscopy and isotopic imaging, time-resolved positron annihilation spectroscopy (PAS), and MeV flash radiography. Indeed, the critical energy for third-generation synchrotron light sources are generally of the order of a few tens of $\mathrm{keV}$, and their output above $100 \mathrm{keV}$ falls off exponentially; fourth-generation light sources such as the planned Linac Coherent Light Source (LCLS) [1,2] are intrinsically lower photon energy machines, as they rely on wigglers with periods in the $\mathrm{cm}$ range to drive the free-electron laser (FEL) [3] instability; finally, for two-stage FELs [4], where the FEL is used as the drive laser for Compton scattering $\gamma$ rays off the storage ring electron beam, optimum electron beam conditions for lasing and Compton scattering are incompatible to a large degree; as a result the peak brightness is considerably smaller than that achievable with an independent drive laser. This is summarized in Fig. 1, where the peak brightness of current light sources is presented as a function of their operating $\mathrm{x}$-ray energy.
In this paper, we consider the merit of Compton scattering [5-13] as a promising alternative approach to develop bright, compact hard $\mathrm{x}$-ray and $\gamma$-ray light sources, whereby the incident photons produced by a picosecond tabletop laser system scatter off a high-brightness, relativistic electron beam to be Doppler-upshifted to energies in the $0.1-10 \mathrm{MeV}$ range. More specifically, the high-energy peak brightness scaling of such Compton scattering light sources is derived, and it is shown that for low normalized emittance electron beams $(1 \mathrm{nC}, 1 \mathrm{~mm}$. $\mathrm{mrad},<1 \mathrm{ps},>100 \mathrm{MeV}$ ), and tabletop laser systems (1-10 J, 5 ps Fourier-transform-limited) the peak x-ray brightness can exceed $10^{23}$ photons $/\left(\mathrm{mm}^{2} \times \operatorname{mrad}^{2} \times \mathrm{s} \times\right.$ $0.1 \%$ bandwidth) near $\omega_{x}=1 \mathrm{MeV}$; this is confirmed by three-dimensional codes $[8,12]$ that have been extensively benchmarked against Compton scattering experiments performed at Lawrence Livermore National Laboratory (LLNL) [11-13]. An analytical expression for the peak on-axis brightness is a powerful tool to efficiently explore the 12-dimensional parameter space corresponding to the phase spaces of both the electron and incident laser beams and to determine optimum conditions for producing highbrightness $\mathrm{x}$ rays. The unique favorable high-energy scaling of Compton x-ray sources is presented in Fig. 1, and shows that a novel region of the peak brightness-energy 


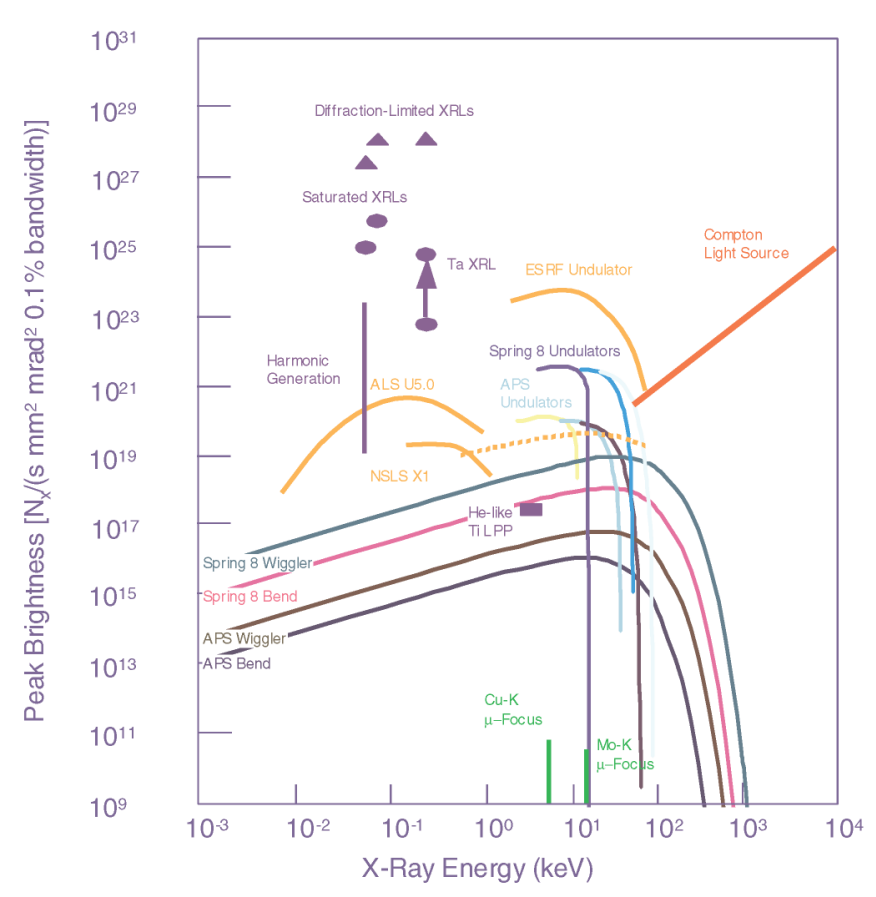

FIG. 1. (Color) Peak brightness of various x-ray sources as a function of their operating photon energy.

chart could be accessed by such new light sources. We also note that the Compton scattering terminology is used throughout this paper in view of the fact that it encompasses a broader parameter range than Thomson scattering, which represents the zero-recoil limit of this elastic scattering process; for a discussion of the conditions where the Thomson limit applies, we refer the reader to Section VI B.

Within the theoretical framework presented here the incident laser pulse is modeled in terms of photons, as opposed to a classical coherent electromagnetic field, and the electrons are described via the Compton scattering differential cross section. The main reason for using a photon model is that it allows the description of the scattered radiation both in the time and frequency domains: if one use the well-known Larmor radiation formula and Fourier-transform analysis, the temporal information is lost, as one integrates over time to perform the Fourier transform yielding the spectral content of the scattered radiation, then squares the result, thus discarding the phase information. The photon description, however, imposes limitations to the modeling of nonlinear effects, where the ponderomotive force plays an important role in modulating the axial dynamics of the particles; some interference phenomena are also neglected.

The interaction geometry under consideration here is head-on collisions, where the $\mathrm{x}$-ray flash duration is shown to be equal to that of the electron bunch, and which produce the highest peak brightness for properly compressed electron beams. Another important aspect of the analysis presented in this paper is the inclusion of nonlinear effects, such as spectral broadening, which are gen- erally ignored in the literature. To our knowledge, this represents the first attempt to model three-dimensional nonlinear effects in Compton scattering. These effects are important, as they show that there is an optimum laser pulse duration in this geometry, of the order of a few picoseconds, in sharp contrast with the initial approach to laser-driven Compton scattering sources where femtosecond laser systems were thought to be mandatory.

This paper is organized as follows: in Sec. II, the temporal x-ray flux and integrated dose are studied; in Sec. III, the peak on-axis brightness of a Compton scattering light source is derived, and an analytical expression is obtained under the assumption that both the electron beam normalized emittance and energy spread remain small; in Sec. IV, nonlinear effects are included; in Sec. V, a brief discussion is presented regarding the parameter space where the analytical theory applies, while Sec. VI focuses on the comparison between the analytical brightness expression derived in Sec. III and a three-dimensional time and frequency-domain code [12] that has been extensively benchmarked against Compton scattering experiments performed at LLNL [11-13], for electron beam and laser parameters corresponding to the current state-of-the art; finally, conclusions are presented in Sec. VII.

\section{FLUX AND X-RAY DOSE}

The local number of $\mathrm{x}$-ray photons scattered per unit time and volume is given by the product of the electron beam 4-current, $j_{\mu}\left(x_{\nu}\right)=e c n_{e}\left(x_{\nu}\right) u_{\mu} / \gamma$, and the incident photon 4-flux, $\Phi_{\mu}\left(x_{\nu}\right)=c n_{\lambda}\left(x_{\nu}\right) k_{\mu} / \omega[14]$ :

$$
\frac{d^{4} N_{x}\left(x_{\nu}\right)}{d^{4} x_{\nu}}=\frac{\sigma}{e c} j_{\mu}\left(x_{\nu}\right) \Phi^{\mu}\left(x_{\nu}\right)=\frac{\sigma c}{\gamma \omega} n_{e}\left(x_{\nu}\right) n_{\lambda}\left(x_{\nu}\right) u_{\mu} k^{\mu} .
$$

Here, $u_{\mu}=d x_{\mu} / c d \tau=(\gamma, \mathbf{u})$ is the electron 4-velocity, $k_{\mu}=(\omega / c, \mathbf{k})$ is the incident photon 4 -wave number, and $\sigma=8 \pi r_{0}^{2} / 3$ is the Compton scattering cross section, expressed in terms of the classical electron radius, $r_{0}=$ $e^{2} / 4 \pi \varepsilon_{0} m_{0} c^{2}=2.8178 \times 10^{-15} \mathrm{~m}$. In Cartesian coordinates, Eq. (1) can be expressed explicitly as

$$
\frac{d^{4} N_{x}(x, y, z, t)}{d x d y d z c d t}=\sigma n_{e}(x, y, z, t) n_{\lambda}(x, y, z, t)\left(1-\beta \cdot \frac{c \mathbf{k}}{\omega}\right) .
$$

Thus, for collinear and cylindrically symmetric beams colliding head-on, as illustrated in Fig. 2, the temporal behavior of the $\mathrm{x}$-ray pulse can be described by considering

$$
\begin{aligned}
\frac{d^{3} N_{x}}{2 \pi r d r d z c d t} & =\sigma\left(1-\boldsymbol{\beta} \cdot \frac{c \mathbf{k}}{\omega}\right) n_{\lambda}(r, z, t) n_{e}(r, z, t) \\
& =\sigma\left(1+\beta_{0}\right) n_{\lambda}(r, z, t) n_{e}(r, z, t),
\end{aligned}
$$

where we have used the average axial velocity of the electron beam, $\boldsymbol{\beta}=\beta_{0} \hat{\mathbf{z}}$, and the average propagation 


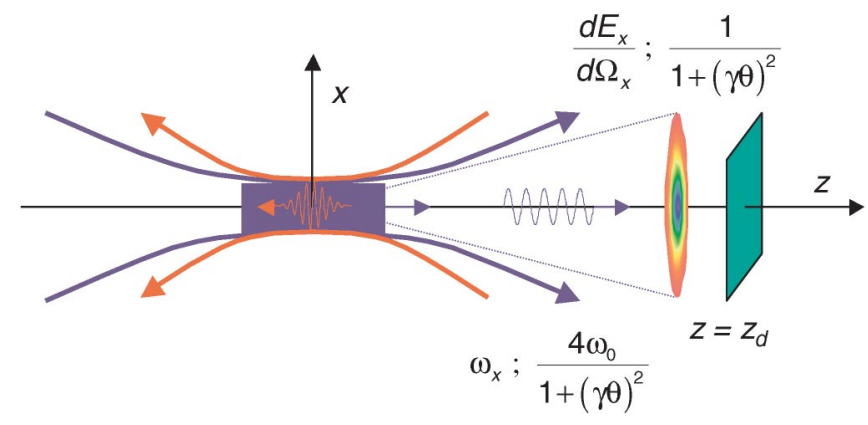

FIG. 2. (Color) Schematic of the interaction geometry, showing the $1 / \gamma$ x-ray energy cone, $d E_{x} / d \Omega_{x}$, the Doppler-upshifted scattered radiation frequency, $\omega_{x}$, and the detector position, $z_{d}$.

direction of the incident laser pulse, $c \mathbf{k} / \omega=-\hat{\mathbf{z}}$, and where spatial positions are given in terms of cylindrical coordinates. Since we are interested in the temporal $x$-ray flux measured by a detector positioned on axis at $z=z_{d}$, we must take into account the retardation condition: the detection time, $t_{d}$, is connected to the emission time and axial position by the relation

$$
t_{d}=t+\frac{z_{d}-z}{c}
$$

mathematically, this is achieved by multiplying Eq. (3) by the delta-function retardation condition, and integrating over time, to obtain

$$
\begin{aligned}
\frac{d^{3} N_{x}}{2 \pi r d r d z d t_{d}}= & \int_{-\infty}^{+\infty} \sigma\left(1+\beta_{0}\right) n_{\lambda}(r, z, t) n_{e}(r, z, t) \\
& \times \delta\left(t_{d}-t+\frac{z_{d}-z}{c}\right) c d t \\
= & c \sigma\left(1+\beta_{0}\right) n_{e \lambda}\left(r, z, t_{d}-\frac{z_{d}-z}{c}\right),
\end{aligned}
$$

where we have introduced the product of the electron and incident photon densities, $n_{e \lambda}=n_{e} n_{\lambda}$. Note that in the left-hand side of Eq. (5), the time, $t$, is now replaced by the detector time, $t_{d}$. In addition, since the temporal x-ray pulse does not depend on the position of the detector, we can set $z_{d}=0$.

Near the focal region, the electron beam density can be modeled by Gaussian radial and temporal distributions

$$
\begin{aligned}
n_{e}(r, z, t)= & \frac{N_{e}}{\sqrt{\pi}^{3} c \Delta \tau r_{b}^{2}} \frac{1}{1+\left(k_{f} z\right)^{2}} \\
& \times \exp \left\{-\left(\frac{t-\left(u_{0} z / \gamma_{0} c\right)}{\Delta \tau}\right)^{2}\right. \\
& \left.-\frac{r^{2}}{r_{b}^{2}\left[1+\left(k_{f} z\right)^{2}\right]}\right\},
\end{aligned}
$$

where $N_{e}=q / e$ is the number of electrons in the bunch, $\Delta \tau$ is the bunch duration, $r_{b}$ is the radius at focus, and where the inverse beta function is given in terms of the normalized emittance, $\varepsilon$, beam focal radius, and energy, $\gamma_{0}$, by $k_{f}=\beta_{f}^{-1}=\varepsilon / \gamma_{0} r_{b}^{2}$. Similarly, the photon density of the focusing and diffracting laser pulse can be described by Gaussian radial and temporal distributions

$$
\begin{aligned}
n_{\lambda}(r, z, t)= & \frac{N_{\lambda}}{\sqrt{\pi / 2}^{3} c \Delta t w_{0}^{2}} \frac{1}{1+\left(z / z_{0}\right)^{2}} \\
& \times \exp \left\{-2\left(\frac{t+(z / c)}{\Delta t}\right)^{2}\right. \\
& \left.-2 \frac{r^{2}}{w_{0}^{2}\left[1+\left(z / z_{0}\right)^{2}\right]}\right\}
\end{aligned}
$$

where $N_{\lambda}=W / \hbar \omega_{0}$ is the total number of photons in the laser pulse, $\Delta t$ is the pulse duration, and is related to the bandwidth as $\Delta t \Delta \omega=\sqrt{2}$, in the case of a Fourier-transform-limited pulse, $w_{0}$ is the $1 / e^{2}$ focal radius, and $z_{0}=$ $\pi w_{0}^{2} / \lambda_{0}$ is the Rayleigh length. The corresponding interaction geometry is illustrated in Fig. 2. Although the Gaussian distributions chosen here do not provide an exhaustive description of the electron and incident photon density distributions, they provide an appropriate model that can be handled analytically. We also note that there is a strong parallel between Eqs. (6) and (7); in particular, the Rayleigh length and the beta function play similar roles, as well as emittance and wavelength; for a more detailed discussion, we refer the reader to [15].

Using the expression of the density product and introducing the normalized axial position, $\bar{z}=2 \sqrt{2} z / c \Delta t$, the normalized inverse beta function, $\eta=k_{f} c \Delta t / 2 \sqrt{2}$, and the normalized inverse Rayleigh length, $\mu=c \Delta t / 2 \sqrt{2} z_{0}$, we have

$$
\begin{aligned}
\frac{d^{3} N_{x}}{2 \pi r d r d \bar{z} d t_{d}}= & \sigma\left(1+\beta_{0}\right) \frac{N_{e} N_{\lambda}}{\pi^{3} r_{b}^{2} w_{0}^{2} \Delta \tau} \\
& \times \frac{1}{\left(1+\eta^{2} \bar{z}^{2}\right)\left(1+\mu^{2} \bar{z}^{2}\right)} \exp \left\{-\left[\frac{t_{d}}{\Delta \tau}\right.\right. \\
& \left.+\bar{z} \frac{\left(1-\beta_{0}\right)}{2 \sqrt{2}} \frac{\Delta t}{\Delta \tau}\right]^{2}-\left(\sqrt{2} \frac{t_{d}}{\Delta t}+\bar{z}\right)^{2} \\
& \left.-\frac{r^{2}}{r_{b}^{2}\left(1+\eta^{2} \bar{z}^{2}\right)}-\frac{2 r^{2}}{w_{0}^{2}\left(1+\mu^{2} \bar{z}^{2}\right)}\right\} .
\end{aligned}
$$

The integral over the radius is easily performed [16]:

$$
\begin{aligned}
& \int_{0}^{\infty} \exp \left\{-r^{2}\left[\frac{1}{r_{b}^{2}\left(1+\eta^{2} \bar{z}^{2}\right)}+\frac{2}{w_{0}^{2}\left(1+\mu^{2} \bar{z}^{2}\right)}\right]\right\} 2 \pi r d r \\
& \quad=\frac{\pi}{\left[1 / r_{b}^{2}\left(1+\eta^{2} \bar{z}^{2}\right)\right]+\left[2 / w_{0}^{2}\left(1+\mu^{2} \bar{z}^{2}\right)\right]}
\end{aligned}
$$

and we find that 


$$
\begin{aligned}
\frac{d N_{x}}{d t_{d}}= & \frac{8}{3 \pi} \frac{r_{0}^{2}}{w_{0}^{2}}\left(1+\beta_{0}\right) \frac{N_{e} N_{\lambda}}{\Delta \tau} \int_{-\infty}^{+\infty} \\
& \times \frac{d \bar{z}}{1+\mu^{2} \bar{z}^{2}+2\left(r_{b}^{2} / w_{0}^{2}\right)\left(1+\eta^{2} \bar{z}^{2}\right)} \\
& \times \exp \left\{-\left[\frac{t_{d}}{\Delta \tau}+\bar{z} \frac{\left(1-\beta_{0}\right)}{2 \sqrt{2}} \frac{\Delta t}{\Delta \tau}\right]^{2}\right. \\
& \left.-\left(\sqrt{2} \frac{t_{d}}{\Delta t}+\bar{z}\right)^{2}\right\} .
\end{aligned}
$$

In the $180^{\circ}$ interaction geometry, this expression can be approximated by

$$
\begin{aligned}
\frac{d N_{x}}{d t_{d}} \simeq & \frac{8}{3 \pi} \frac{r_{0}^{2}}{w_{0}^{2}}\left(1+\beta_{0}\right) \frac{N_{e} N_{\lambda}}{\Delta \tau} \exp \left[-\left(\frac{t_{d}}{\Delta \tau}\right)^{2}\right] \\
& \times \int_{-\infty}^{+\infty} \frac{d \bar{z}}{1+\mu^{2} \bar{z}^{2}+2\left(r_{b}^{2} / w_{0}^{2}\right)\left(1+\eta^{2} \bar{z}^{2}\right)} \\
& \times \exp \left\{-\left[\bar{z} \frac{\left(1-\beta_{0}\right)}{2 \sqrt{2}} \frac{\Delta t}{\Delta \tau}\right]^{2}-\bar{z}^{2}\right\} \\
\simeq & \frac{8}{3 \pi} \frac{r_{0}^{2}}{w_{0}^{2}}\left(1+\beta_{0}\right) \frac{N_{e} N_{\lambda}}{\Delta \tau} \exp \left[-\left(\frac{t_{d}}{\Delta \tau}\right)^{2}\right] \\
& \times \int_{-\infty}^{+\infty} \frac{e^{-\bar{z}^{2}} d \bar{z}}{1+\mu^{2} \bar{z}^{2}+2\left(r_{b}^{2} / w_{0}^{2}\right)\left(1+\eta^{2} \bar{z}^{2}\right)},
\end{aligned}
$$

and the integral can be performed, using the fact that [16]

$$
\int_{0}^{\infty} \frac{e^{-x^{2}} d x}{x^{2}+\beta^{2}}=\frac{\pi}{2 \beta} e^{\beta^{2}}[1-\Phi(\beta)]
$$

where $\Phi(x)=(2 / \sqrt{\pi}) \int_{0}^{x} e^{-t^{2}} d t$ is the error function; we then find that

$$
\begin{aligned}
\frac{d N_{x}}{d t_{d}} \simeq & \frac{8}{3} \frac{r_{0}^{2}}{w_{0}^{2}}\left(1+\beta_{0}\right) \frac{N_{e} N_{\lambda}}{\Delta \tau} \exp \left[-\left(\frac{t_{d}}{\Delta \tau}\right)^{2}\right] \\
& \times \frac{\left.\left\{1-\Phi\left[\sqrt{\left(1+2 \frac{r_{b}^{2}}{w_{0}^{2}}\right) /\left(\mu^{2}+2 \frac{r_{b}^{2}}{w_{0}^{2}} \eta^{2}\right.}\right)\right]\right\}}{\sqrt{\left(\mu^{2}+2 \frac{r_{b}^{2}}{w_{0}^{2}} \eta^{2}\right)\left(1+2 \frac{r_{b}^{2}}{w_{0}^{2}}\right)}} \\
& \times \exp \left(\frac{1+2 \frac{r_{b}^{2}}{w_{0}^{2}}}{\mu^{2}+2 \frac{r_{b}^{2}}{w_{0}^{2}} \eta^{2}}\right),
\end{aligned}
$$

which can be written in a more compact form by noting that

$$
2 \frac{r_{b}^{2}}{w_{0}^{2}}=\frac{2 \pi \varepsilon}{\lambda_{0} \gamma_{0}} \frac{\mu}{\eta}=\xi \frac{\mu}{\eta}
$$

and introducing the overlap function,

$$
\begin{aligned}
\mathcal{F}(\xi, \eta, \mu)= & \frac{\left\{1-\Phi\left[\sqrt{(\eta+\xi \mu) /\left(\eta \mu^{2}+\xi \mu \eta^{2}\right)}\right]\right\}}{\sqrt{\left(\mu^{2}+\xi \mu \eta\right)\left(1+\xi \frac{\mu}{\eta}\right)}} \\
& \times \exp \left(\frac{\eta+\xi \mu}{\eta \mu^{2}+\xi \mu \eta^{2}}\right)
\end{aligned}
$$

to obtain

$$
\begin{aligned}
\frac{d N_{x}}{d t_{d}} & \simeq \frac{8}{3} \frac{r_{0}^{2}}{w_{0}^{2}}\left(1+\beta_{0}\right) \frac{N_{e} N_{\lambda}}{\Delta \tau} \mathcal{F}(\xi, \eta, \mu) \exp \left[-\left(\frac{t_{d}}{\Delta \tau}\right)^{2}\right] \\
& =\frac{N_{x}}{\sqrt{\pi} \Delta \tau} e^{-t_{d}^{2} / \Delta \tau^{2}} .
\end{aligned}
$$

The behavior of the overlap function is illustrated in Fig. 3, for a fixed value of the ratio of the electron beam physical emittance, which is defined as the ratio of the normalized emittance to the normalized electron beam energy, $\varepsilon / \gamma$; and laser wavelength, $\xi=2 \pi \varepsilon / \lambda_{0} \gamma_{0}=0.127$, and shows that for laser pulses much longer than the diffraction length, where $\mu \geq 10$, there is no optimal value of the inverse beta function that yields a good overlap; for laser pulses dominated by diffraction the optimum lies roughly along the $\mu \eta \approx 1 / \xi$ region: for example, when $\mu=1$, the maximum value of $\mathcal{F}$ is reached when $\eta \simeq 10$, while for $\mu=0.1, \eta \simeq 100$ is approximately optimum.

Finally, the total dose can easily be derived by integrating Eq. (16) over the detection time:

$$
N_{x} \simeq \frac{8 \sqrt{\pi}}{3} \frac{r_{0}^{2}}{w_{0}^{2}}\left(1+\beta_{0}\right) N_{e} N_{\lambda} \mathcal{F}(\xi, \eta, \mu) .
$$

The validity of the approximation used above is shown in Fig. 4, where both the integral in Eq. (10), shown as blue squares and blue dotted line, and the approximation in Eq. (13), shown as a red line, are shown to be in excellent agreement for the specific parameters discussed in the

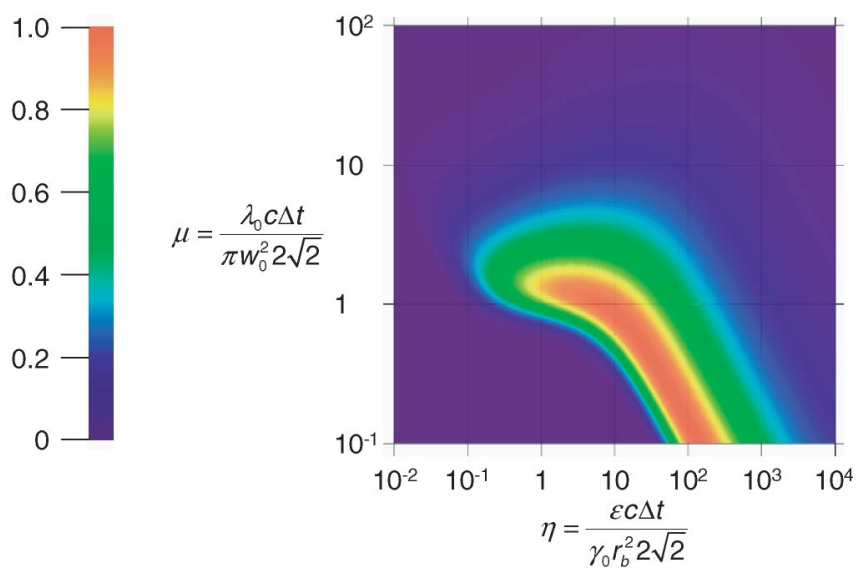

FIG. 3. (Color) Overlap function, $\mathcal{F}$, as a function of the normalized inverse beta function, $\eta=\beta_{f} c \Delta t / 2 \sqrt{2}$, and normalized diffraction length, $\mu=c \Delta t / z_{0} 2 \sqrt{2}$, for a fixed value of $\xi=$ $2 \pi \varepsilon / \lambda_{0} \gamma_{0}=0.127$. The color scale is linear, with arbitrary units. 


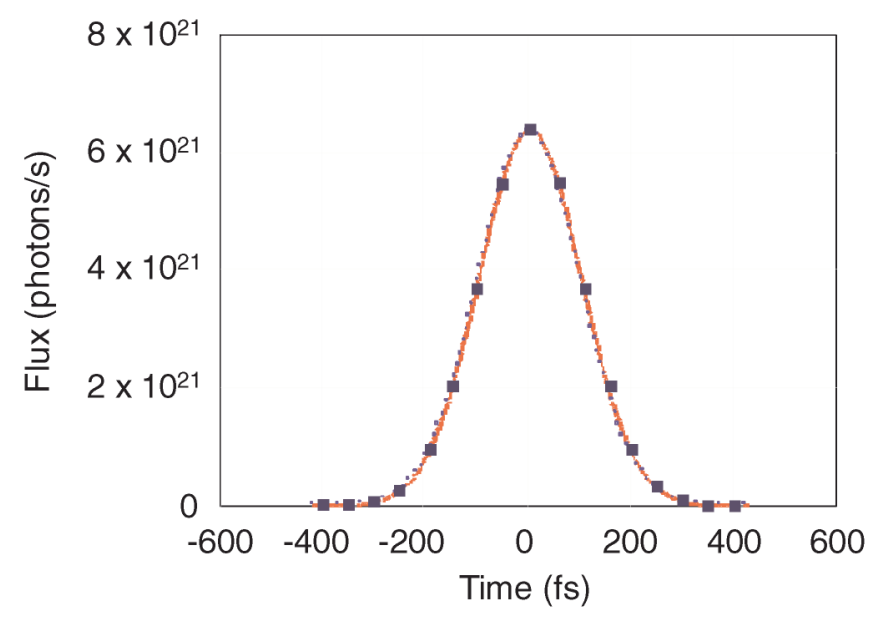

FIG. 4. (Color) Temporal evolution of the x-ray flux for a $1 \mathrm{nC}$, $\Delta \tau / \sqrt{2}=100 \mathrm{fs}$ rms, $50 \mathrm{MeV}$ compressed electron bunch colliding with a $1 \mathrm{~J}, 1 \mu \mathrm{m}, 5 \mathrm{ps}$ FTL laser pulse. The electron beam focal radius is $r_{b} / \sqrt{2}=10 \mu \mathrm{m} \mathrm{rms}$, while the laser $1 / e^{2}$ intensity focal radius is $w_{0}=20 \mu \mathrm{m}$. The integrated dose is $N_{x}=$ $1.6 \times 10^{9}$ photons, and the corresponding quantum efficiency is 0.256 photon/electron.

caption; in general, excellent agreement is obtained over a wide parameter range. This is an important result, because it demonstrates that in the $180^{\circ}$ interaction geometry, the temporal $\mathrm{x}$-ray pulse profile closely follows the electron beam pulse shape. Physically, this result can be understood as follows: if the laser pulse is longer than the electron bunch, then the situation is that of an electromagnetic wiggler, which is essentially analogous to a magnetostatic wiggler, where the pulse duration is governed by the electron beam duration (see, for example, LCLS references $[1,2])$; in the case when the laser pulse is shorter than the electron beam, the electrons still wiggle under the influence of the laser, and as the $\mathrm{x}$ rays propagate at the speed of light, while the electrons are highly relativistic, the respective pulse durations remain very similar. For other interaction geometries, however, this is no longer true: in particular, at $90^{\circ}$, for $\Delta \tau \gg \Delta t$, the $\mathrm{x}$-ray pulse duration is governed by the transit time of the laser pulse across the electron beam focus.

\section{PEAK BRIGHTNESS SCALING}

As indicated above, the number of $\mathrm{x}$-ray photons radiated per unit volume and per unit time is given by

$$
\begin{aligned}
\frac{d^{4} N_{x}}{d^{4} x_{\mu}} & =\sigma c \frac{u_{\nu} k^{\nu}}{\gamma \omega} n_{\lambda}\left(x_{\mu}\right) n_{e}\left(x_{\mu}\right) \\
& =\sigma\left(1-\boldsymbol{\beta} \cdot \frac{c \mathbf{k}}{\omega}\right) n_{\lambda}\left(x_{\mu}\right) n_{e}\left(x_{\mu}\right) .
\end{aligned}
$$

To obtain the radiated x-ray density per unit solid angle, one can use the differential scattering cross section:

$$
\frac{d^{5} N_{x}}{d^{4} x_{\mu} d \Omega_{x}}=\frac{d \sigma}{d \Omega_{x}} c \frac{u_{\nu} k^{\nu}}{\gamma \omega} n_{\lambda}\left(x_{\mu}\right) n_{e}\left(x_{\mu}\right)
$$

Finally, the local spectral brightness can be derived by using the Doppler-shifted x-ray frequency, which is given by

$$
\frac{\omega_{x}}{\omega}=\frac{\kappa}{\kappa_{x}}=\frac{\gamma-\mathbf{u} \cdot \frac{c \mathbf{k}}{\omega}}{\gamma-\mathbf{u} \cdot \hat{\mathbf{n}}_{x}}
$$

in the Thomson limit; here, $\hat{\mathbf{n}}_{x}$ is the direction of observation. It is easily seen that for head-on collisions, the frequency of the backscattered radiation is Doppler upshifted by approximately $4 \gamma^{2}$ : using $c \mathbf{k} / \omega=-\hat{\mathbf{z}}$ and $\hat{\mathbf{n}}_{x}=\hat{\mathbf{z}}$, $\omega_{x}=\omega\left(\gamma+u_{z}\right) /\left(\gamma-u_{z}\right) \simeq 4 \gamma^{2} \omega$. We then have

$$
\begin{aligned}
\frac{d^{6} N_{x}}{d^{4} x_{\mu} d \Omega_{x} d \omega_{x}}= & \frac{d \sigma}{d \Omega_{x}}\left(1-\boldsymbol{\beta} \cdot \frac{c \mathbf{k}}{\omega}\right) n_{\lambda}\left(x_{\mu}\right) n_{e}\left(x_{\mu}\right) \\
& \times \delta\left(\omega_{x}-\omega \frac{\kappa}{\kappa_{x}}\right)
\end{aligned}
$$

where $\kappa$ and $\kappa_{x}$ are the electron light-cone variables with respect to the incident and scattered photons, respectively, as defined in Eq. (20).

For realistic electron and laser beams, Eq. (21) must be integrated over the entire phase space of each beam to yield the x-ray brightness, within the context of an incoherent superposition.

We now consider a drive laser pulse with uncorrelated Gaussian phase space; in the lab frame, the laser propagates along the $z$ axis in the negative direction, while the linear polarization lies in the $x$ - $z$ plane. In addition, the electron beam propagates along the positive $z$ axis, which corresponds to head-on collisions at $180^{\circ}$; finally, we choose the direction of observation to lie along the electron beam axis, where the brightness is highest, by letting $\hat{\mathbf{n}}_{x}=\hat{\mathbf{z}}$.

For this specific interaction geometry, illustrated in Fig. 2, and neglecting the transverse laser wave number spectrum, which only adds very small biquadratic correction terms for extremely tight laser foci, the differential scattering cross section takes a very simple form [12]:

$$
\left.\frac{d \sigma}{d \Omega_{x}}\right|_{\hat{\mathbf{n}}_{x}=\hat{\mathbf{z}}}=r_{0}^{2}\left(\frac{\gamma+u}{\gamma-u}\right)
$$

and the local on-axis spectral brightness reads

$$
\begin{aligned}
\left.\frac{d^{6} N_{x}}{d^{4} x_{\mu} d \Omega_{x} d \omega_{x}}\right|_{\hat{\mathbf{n}}_{x}=\hat{\mathbf{z}}}= & r_{0}^{2}\left(\frac{\gamma+u}{\gamma-u}\right)(1 \\
& \left.+\beta_{z}\right) n_{\lambda}\left(x_{\mu}\right) n_{e}\left(x_{\mu}\right) \delta\left(\omega_{x}-\omega \frac{\kappa}{\kappa_{x}}\right) .
\end{aligned}
$$




\section{A. Laser spectrum}

Integrating over the laser spectrum, to account for the incident photon energy distribution, we now have

$$
\begin{aligned}
S_{\omega}= & \left.\frac{1}{\sqrt{\pi} \Delta \omega} \int_{-\infty}^{+\infty} \frac{d^{6} N_{x}}{d^{4} x_{\mu} d \Omega_{x} d \omega_{x}}\right|_{\hat{\mathbf{n}}_{x}=\hat{\mathbf{z}}} \\
& \times \exp \left[-\left(\frac{\omega-\omega_{0}}{\Delta \omega}\right)^{2}\right] d \omega \\
= & r_{0}^{2}\left(\frac{\gamma+u}{\gamma-u}\right)\left(1+\beta_{z}\right) n_{\lambda}\left(x_{\mu}\right) n_{e}\left(x_{\mu}\right) \frac{1}{\sqrt{\pi} \Delta \omega} \int_{-\infty}^{+\infty} \\
& \times \delta\left(\omega_{x}-\omega \frac{\gamma+u_{z}}{\gamma-u_{z}}\right) \exp \left[-\left(\frac{\omega-\omega_{0}}{\Delta \omega}\right)^{2}\right] d \omega .
\end{aligned}
$$

The integral in Eq. (24) is easily performed [16], with the result that

$$
\begin{aligned}
S_{\omega}= & r_{0}^{2}\left(\frac{\gamma+u}{\gamma-u}\right)\left(1+\beta_{z}\right) n_{\lambda}\left(x_{\mu}\right) n_{e}\left(x_{\mu}\right) \frac{1}{\sqrt{\pi} \Delta \omega}\left(\frac{\gamma-u_{z}}{\gamma+u_{z}}\right) \\
& \times \exp \left\{-\left[\frac{\omega_{x}\left[\left(\gamma-u_{z}\right) /\left(\gamma+u_{z}\right)\right]-\omega_{0}}{\Delta \omega}\right]^{2}\right\}
\end{aligned}
$$

\section{B. Energy spread}

Next, the effects of energy spread can be considered by integrating the local on-axis brightness over a Gaussian energy distribution:

$$
S_{\gamma}=\frac{\int_{1}^{\infty} S_{\omega}(\gamma) \exp \left\{-\left[\left(\gamma-\gamma_{0}\right) / \Delta \gamma\right]^{2}\right\} d \gamma}{\int_{1}^{\infty} \exp \left\{-\left[\left(\gamma-\gamma_{0}\right) / \Delta \gamma\right]^{2}\right\} d \gamma} .
$$

It proves convenient and sufficiently accurate to replace the lower integral bound by $-\infty$; in this case the normalization factor is $1 / \sqrt{\pi} \Delta \gamma$, and Eq. (26) becomes

$$
\begin{aligned}
S_{\gamma} \simeq & \frac{r_{0}^{2} n_{e} n_{\lambda}}{\sqrt{\pi} \Delta \omega} \frac{1}{\sqrt{\pi} \Delta \gamma} \int_{-\infty}^{+\infty}\left(\frac{\gamma+u}{\gamma-u}\right)\left(\frac{\gamma-u_{z}}{\gamma}\right) \\
& \times \exp \left\{-\left[\frac{\omega_{x}\left[\left(\gamma-u_{z}\right) /\left(\gamma+u_{z}\right)\right]-\omega_{0}}{\Delta \omega}\right]^{2}\right. \\
& \left.-\left(\frac{\gamma-\gamma_{0}}{\Delta \gamma}\right)^{2}\right\} d \gamma .
\end{aligned}
$$

At this point, one can use the fact that, for highly relativistic beams, $\gamma^{-1} \ll 1$, and for sufficiently low normalized emittance the transverse electron velocity is much smaller than its axial counterpart, with $u_{\perp} \ll u_{z}$. Within this context, and keeping in mind the fact that

$$
\begin{aligned}
\gamma^{2} & =1+u^{2}=1+u_{\perp}^{2}+u_{z}^{2}, \\
\gamma^{2}-u^{2} & =(\gamma-u)(\gamma+u)=1, \\
\gamma^{2}-u_{z}^{2} & =\left(\gamma-u_{z}\right)\left(\gamma+u_{z}\right)=1+u_{\perp}^{2},
\end{aligned}
$$

we have

$$
\begin{aligned}
\frac{\gamma-u_{z}}{\gamma+u_{z}} & \simeq \frac{1+u_{\perp}^{2}}{4 \gamma^{2}} \\
\left(\frac{\gamma+u}{\gamma-u}\right)\left(\frac{\gamma-u_{z}}{\gamma}\right) & =\frac{(\gamma+u)^{2}}{\gamma\left(\gamma+u_{z}\right)}\left(1+u_{\perp}^{2}\right) \simeq 2\left(1+u_{\perp}^{2}\right) .
\end{aligned}
$$

Furthermore, one can assume that the electron beam energy spread is small compared to the average energy, with $\Delta \gamma / \gamma_{0} \ll 1$, and we can make the following approximation:

$$
\frac{1}{\gamma^{2}}=\left(\gamma_{0}+\delta\right)^{-2} \simeq \frac{1}{\gamma_{0}^{2}}\left(1-2 \frac{\delta}{\gamma_{0}}\right)
$$

where we have introduced the variable $\delta=\gamma-\gamma_{0}$. With this, Eq. (27) takes the simpler form

$$
\begin{aligned}
S_{\gamma} \simeq & \frac{r_{0}^{2} n_{e} n_{\lambda}}{\sqrt{\pi} \Delta \omega} \frac{2\left(1+u_{\perp}^{2}\right)}{\sqrt{\pi} \Delta \gamma} \int_{-\infty}^{+\infty} \\
& \times \exp \left\{-\left[\frac{\left(\omega_{x} / 4 \gamma_{0}^{2}\right)\left(1+u_{\perp}^{2}\right)\left\{1-\left[2\left(\delta / \gamma_{0}\right)\right]\right\}-\omega_{0}}{\Delta \omega}\right]^{2}\right. \\
& \left.-\frac{\delta^{2}}{\Delta \gamma^{2}}\right\} d \delta
\end{aligned}
$$

It proves useful to introduce the normalized Dopplerupshifted frequency, $\chi=\omega_{x} / 4 \gamma_{0}^{2} \omega_{0}$, and the relative spectral width of the drive laser pulse, $\delta \omega=\Delta \omega / \omega_{0}$, to obtain

$$
\begin{aligned}
S_{\gamma} \simeq & \frac{r_{0}^{2} n_{e} n_{\lambda}}{\sqrt{\pi} \Delta \omega} \frac{2\left(1+u_{\perp}^{2}\right)}{\sqrt{\pi} \Delta \gamma} \int_{-\infty}^{+\infty} \\
& \times \exp \left\{-\left[\frac{\chi\left(1+u_{\perp}^{2}\right)\left\{1-\left[2\left(\delta / \gamma_{0}\right)\right]\right\}-1}{\delta \omega}\right]^{2}-\frac{\delta^{2}}{\Delta \gamma^{2}}\right\} d \delta .
\end{aligned}
$$

This integral can be performed analytically [16]:

$$
\int_{-\infty}^{+\infty} \exp \left(-p^{2} x^{2} \pm q x\right) d x=\frac{\sqrt{\pi}}{p} \exp \left(\frac{q^{2}}{4 p^{2}}\right)
$$

introducing the scaled relative energy spread, $\delta \gamma=$ $2 \Delta \gamma / \gamma_{0}$, and identifying terms, we have

$$
\begin{aligned}
& \delta \equiv x, \quad p^{2} \equiv \frac{1}{\Delta \gamma^{2}}\left[1+\frac{\delta \gamma^{2}}{\delta \omega^{2}} \chi^{2}\left(1+u_{\perp}^{2}\right)^{2}\right] \\
& q \equiv \frac{1}{\Delta \gamma}\left\{2 \frac{\delta \gamma}{\delta \omega^{2}} \chi\left(1+u_{\perp}^{2}\right)\left[\chi\left(1+u_{\perp}^{2}\right)-1\right]\right\}
\end{aligned}
$$


after some algebra, we find that

$$
\begin{aligned}
S_{\gamma} \simeq & \frac{2 r_{0}^{2} n_{e} n_{\lambda}}{\sqrt{\pi} \omega_{0}} \frac{1+u_{\perp}^{2}}{\sqrt{\delta \omega^{2}+\delta \gamma^{2} \chi^{2}\left(1+u_{\perp}^{2}\right)^{2}}} \\
& \times \exp \left\{-\frac{\left[\chi\left(1+u_{\perp}^{2}\right)-1\right]^{2}}{\delta \omega^{2}+\delta \gamma^{2} \chi^{2}\left(1+u_{\perp}^{2}\right)^{2}}\right\}
\end{aligned}
$$

\section{Emittance}

The next important aspect of the electron beam phase space is the normalized emittance contribution to the brightness degradation; assuming a cylindrically symmetric beam, both in real and in momentum space, emittance can be modeled by a Gaussian distribution of transverse velocity:

$$
S_{\varepsilon}=\frac{1}{\pi \Delta u_{\perp}^{2}} \int_{0}^{\infty} S_{\gamma}\left(u_{\perp}\right) \exp \left[-\left(\frac{u_{\perp}}{\Delta u_{\perp}}\right)^{2}\right] 2 \pi u_{\perp} d u_{\perp} .
$$

More explicitly, the local on-axis brightness, averaged over the 6-dimensional electron beam phase space, takes the form

$$
\begin{aligned}
& \left\langle\left.\frac{d^{6} N_{x}}{d^{4} x_{\mu} d \Omega_{x} d \omega_{x}}\right|_{\hat{\mathbf{n}}_{x}=\hat{\mathbf{z}}}\right\rangle \\
& =\frac{2 r_{0}^{2}}{\sqrt{\pi} \omega_{0}} n_{e}\left(x_{\mu}\right) n_{\lambda}\left(x_{\mu}\right) S_{\varepsilon}\left(\chi, \delta \omega, \delta \gamma, \Delta u_{\perp}\right),
\end{aligned}
$$

where the normalized spectral brightness is defined as $S_{\varepsilon}=S_{\varepsilon} \sqrt{\pi} \omega_{0} / 2 r_{0}^{2} n_{e} n_{\lambda}:$

$$
\begin{aligned}
S_{\varepsilon}\left(\chi, \delta \omega, \delta \gamma, \Delta u_{\perp}\right)= & \frac{1}{\Delta u_{\perp}^{2}} \int_{0}^{\infty} \\
& \times \frac{2 u_{\perp}\left(1+u_{\perp}^{2}\right)}{\sqrt{\delta \omega^{2}+\delta \gamma^{2} \chi^{2}\left(1+u_{\perp}^{2}\right)^{2}}} \\
& \times \exp \left\{-\frac{\left[\chi\left(1+u_{\perp}^{2}\right)-1\right]^{2}}{\delta \omega^{2}+\delta \gamma^{2} \chi^{2}\left(1+u_{\perp}^{2}\right)^{2}}\right. \\
& \left.-\frac{u_{\perp}^{2}}{\Delta u_{\perp}^{2}}\right\} d u_{\perp} .
\end{aligned}
$$

\section{Time-dependent x-ray brightness}

To obtain the brightness in synchrotron units, namely photons $/\left(\mathrm{mm}^{2} \times \mathrm{mrad}^{2} \times \mathrm{s} \times 0.1 \%\right.$ bandwidth $)$, one must take into account the correlation between the spatial position along the direction of observation of the $x$ rays and the radiation time to translate these variables in terms of the detector time, $t_{d}$, by taking into account the retardation condition, as discussed in Sec. II. Since $\hat{\mathbf{n}}_{x}=\hat{\mathbf{z}}$; we have

$$
\begin{aligned}
\frac{d^{6} N_{x}}{d x d y d z d t_{d} d \Omega_{x} d \omega_{x}}= & \int_{-\infty}^{+\infty} \frac{d^{6} N_{x}}{d^{4} x_{\mu} d \Omega_{x} d \omega_{x}} \\
& \times \delta\left(t_{d}-t-\frac{z_{d}-z}{c}\right) c d t,
\end{aligned}
$$

where $z_{d}$ represents the detector position, and where we have omitted the averaging brackets and the direction of observation for simplicity. We obtain

$$
\begin{aligned}
\frac{d^{6} N_{x}}{d x d y d t d t_{d} d \Omega_{x} d \omega_{x}}= & \frac{c d^{6} N_{x}}{d^{4} x_{\mu} d \Omega_{x} d \omega_{x}}\left(x, y, z, t_{d}-\frac{z_{d}-z}{c}\right) \\
= & \frac{2 r_{0}^{2} c}{\sqrt{\pi} \omega_{0}} S_{\varepsilon}\left(\chi, \delta \omega, \delta \gamma, \Delta u_{\perp}\right) n_{e \lambda} \\
& \times\left(x, y, z, t_{d}-\frac{z_{d}-z}{c}\right),
\end{aligned}
$$

where we have used the density product function $n_{e \lambda}\left(x_{\mu}\right)=n_{e}\left(x_{\mu}\right) n_{\lambda}\left(x_{\mu}\right)$. A second integral yields the sought-after on-axis brightness:

$$
\begin{aligned}
\frac{d^{5} N_{x}\left(t_{d}\right)}{d x d y d t_{d} d \Omega_{x} d \omega_{x}}= & \int_{-\infty}^{+\infty} \frac{d^{6} N_{x}}{d x d y d t d t_{d} d \Omega_{x} d \omega_{x}} d z \\
= & \frac{2 r_{0}^{2} c}{\sqrt{\pi} \omega_{0}} S_{\varepsilon}\left(\chi, \delta \omega, \delta \gamma, \Delta u_{\perp}\right) \\
& \times \int_{-\infty}^{+\infty} n_{e \lambda}\left(0,0, z, t_{d}+\frac{z}{c}\right) d z
\end{aligned}
$$

Note that in Eq. (41), we have set $z_{d}=0$, since the brightness does not depend on the position of the detector, and we have chosen $x=y=0$, since the maximum density product is obtained on-axis in the cases where the electron beam and laser pulse have zero transverse offset.

To translate this quantity into more familiar units, we multiply it by a small surface element, $\delta \Sigma=1 \mathrm{~mm}^{2}=$ $10^{-6} \mathrm{~m}^{2}$, a small solid angle, $\delta \Omega_{x}=1 \mathrm{mrad}^{2}=$ $10^{-6} \mathrm{rad}^{2}$, and by $0.1 \%$ fractional bandwidth, $\delta \omega_{x}=$ $10^{-3} \omega_{x}$ :

$$
\begin{aligned}
B_{x} & =\frac{d^{5} N_{x}}{d x d y d t_{d} d \Omega_{x} d \omega_{x}} \times \delta \Sigma \times \delta \Omega_{x} \times \delta \omega_{x} \\
& =\frac{d^{5} N_{x}}{d x d y d t_{d} d \Omega_{x} d \omega_{x}} \times 10^{-15} \times \omega_{x} .
\end{aligned}
$$

Using the definition of the normalized Dopplerupshifted frequency, we can recast Eq. (42) to obtain the peak, on-axis brightness:

$$
\begin{aligned}
\hat{B}_{x}= & \frac{8 \times 10^{-15} \gamma_{0}^{2} r_{0}^{2} c \chi}{\sqrt{\pi}} S_{\varepsilon}\left(\chi, \delta \omega, \delta \gamma, \Delta u_{\perp}\right) \\
& \times \int_{-\infty}^{+\infty} n_{e \lambda}\left(r=0, z, \frac{z}{c}\right) d z
\end{aligned}
$$

where we have assumed that there is no temporal offset 
between the laser pulse and the electron bunch, so that the peak brightness occurs for $t_{d}=0$, when $z_{d}=0$.

\section{E. Analytical peak brightness expression}

Further simplifications can be obtained in the case where the main degradation due to the electron beam emittance is a low-energy tail in the x-ray spectrum caused by the tilt of the $\mathrm{x}$-ray cones emitted by electrons crossing the axis with a small, but nonzero angle; in that case Eq. (38) can be approximated as follows:

$$
\begin{aligned}
S_{\varepsilon}\left(\chi, \delta \omega, \delta \gamma, \Delta u_{\perp}\right) & =\frac{1}{\Delta u_{\perp}^{2}} \int_{0}^{\infty} \frac{(1+x)}{\sqrt{\delta \omega^{2}+\delta \gamma^{2} \chi^{2}(1+x)^{2}}} \exp \left\{-\frac{[\chi(1+x)-1]^{2}}{\delta \omega^{2}+\delta \gamma^{2} \chi^{2}(1+x)^{2}}-\frac{x}{\Delta u_{\perp}^{2}}\right\} d x, \\
& \simeq \frac{1}{\Delta u_{\perp}^{2}} \frac{1}{\sqrt{\delta \omega^{2}+\delta \gamma^{2} \chi^{2}}} \int_{0}^{\infty} \exp \left\{-\frac{[\chi(1+x)-1]^{2}}{\delta \omega^{2}+\delta \gamma^{2} \chi^{2}}-\frac{x}{\Delta u_{\perp}^{2}}\right\} d x .
\end{aligned}
$$

Here, we have first changed variables by using $x=u_{\perp}^{2}$, and kept only the lowest-order spectral dependence on $u_{\perp}^{2}$. Equation (44) can be integrated analytically [16]:

$$
\int_{0}^{\infty} \exp \left(-\frac{x^{2}}{4 \beta}-\gamma x\right) d x=\sqrt{\pi \beta} \exp \left(\beta \gamma^{2}\right)[1-\Phi(\gamma \sqrt{\beta})]
$$

Identifying terms, we find that

$$
S_{\varepsilon}\left(\chi, \delta \omega, \delta \gamma, \Delta u_{\perp}\right) \simeq \frac{\sqrt{\pi}}{2 \chi \Delta u_{\perp}^{2}} \exp \left\{\frac{\chi-1}{2 \chi \Delta u_{\perp}^{2}}\left[2+\frac{\delta \omega^{2}+\delta \gamma^{2} \chi^{2}}{2 \chi(\chi-1) \Delta u_{\perp}^{2}}\right]\right\}\left[1-\Phi\left\{\frac{\chi-1}{\sqrt{\delta \omega^{2}+\delta \gamma^{2} \chi^{2}}}\left[1+\frac{\delta \omega^{2}+\delta \gamma^{2} \chi^{2}}{2 \chi(\chi-1) \Delta u_{\perp}^{2}}\right]\right\}\right] .
$$

Furthermore, the axial overlap integral in Eq. (43) can also be expressed analytically: as previously indicated, the electron beam density is given by Eq. (6), and the incident photon density of the focusing and diffracting laser pulse is described by Eq. (7). When the beams are collinear, and have no temporal or radial offsets, we have

$$
\int_{-\infty}^{+\infty} n_{e \lambda}\left(r=0, z, \frac{z}{c}\right) d z=\frac{N_{e} N_{\lambda}}{(\pi / \sqrt{2})^{3} c^{2} r_{b}^{2} w_{0}^{2} \Delta \tau \Delta t} \int_{-\infty}^{+\infty} \frac{d z}{\left[1+\left(k_{f} z\right)^{2}\right]\left[1+\left(z / z_{0}\right)^{2}\right]} \exp \left\{-\frac{z^{2}}{c^{2}}\left[\frac{\left[1-\left(u_{0} / \gamma_{0}\right)^{2}\right.}{\Delta \tau^{2}}+\frac{8}{\Delta t^{2}}\right]\right\} .
$$

At this point, we note that for relativistic beams,

$$
\frac{\left[1-\left(u_{0} / \gamma_{0}\right)\right]^{2}}{\Delta \tau^{2}}+\frac{8}{\Delta t^{2}} \simeq \frac{1}{4 \gamma_{0}^{4} \Delta \tau^{2}}+\frac{8}{\Delta t^{2}} \simeq \frac{8}{\Delta t^{2}},
$$

where the last approximation stands for most practical cases. Within those circumstances, Eq. (47) can be integrated analytically [17]:

$$
\begin{aligned}
& \frac{N_{e} N_{\lambda}}{(\pi / \sqrt{2})^{3} c^{2} r_{b}^{2} w_{0}^{2} \Delta \tau \Delta t} \int_{-\infty}^{+\infty} \frac{d z}{\left[1+\left(k_{f} z\right)^{2}\right]\left[1+\left(z / z_{0}\right)^{2}\right]^{2}} \exp \left(-\frac{8 z^{2}}{c^{2} \Delta t^{2}}\right)=\frac{N_{e} N_{\lambda}}{\pi^{3} c r_{b}^{2} w_{0}^{2} \Delta \tau} \int_{-\infty}^{+\infty} \frac{e^{-\bar{z}^{2}} d \bar{z}}{\left(1+\eta^{2} \bar{z}^{2}\right)\left(1+\mu^{2} \bar{z}^{2}\right)} \\
& =\frac{N_{e} N_{\lambda}}{\pi^{2} c r_{b}^{2} w_{0}^{2} \Delta \tau} \frac{\eta e^{1 / \eta^{2}[\Phi(1 / \eta)-1]-\mu e^{1 / \mu^{2}}[\Phi(1 / \mu)-1]}}{\mu^{2}-\eta^{2}}=\frac{N_{e} N_{\lambda}}{\pi^{3} c r_{b}^{2} w_{0}^{2} \Delta \tau} \mathcal{O}(\eta, \mu),
\end{aligned}
$$

where we have used the normalized axial position, $\bar{z}=2 \sqrt{2} z / c \Delta t$, and the dimensionless beam envelope parameters defined in Sec. II.

Using these results in the expression of the peak brightness, we finally find that

$$
\begin{aligned}
\hat{B}_{x}= & \frac{4 \times 10^{-15}}{\pi^{2}} \frac{\gamma_{0}^{2}}{\varepsilon^{2}} \frac{N_{e} N_{\lambda}}{\Delta \tau} \frac{r_{0}^{2}}{w_{0}^{2}} \exp \left\{\frac{\chi-1}{2 \chi \Delta u_{\perp}^{2}}\left[2+\frac{\delta \omega^{2}+\delta \gamma^{2} \chi^{2}}{2 \chi(\chi-1) \Delta u_{\perp}^{2}}\right]\right\}\left[1-\Phi\left\{\frac{\chi-1}{\sqrt{\delta \omega^{2}+\delta \gamma^{2} \chi^{2}}}\left[1+\frac{\delta \omega^{2}+\delta \gamma^{2} \chi^{2}}{2 \chi(\chi-1) \Delta u_{\perp}^{2}}\right]\right\}\right] \\
& \times \frac{\eta e^{1 / \mu^{2}}[\Phi(1 / \eta)-1]-\mu e^{1 / \mu^{2}}[\Phi(1 / \mu)-1]}{\mu^{2}-\eta^{2}}
\end{aligned}
$$

where we have made the approximation $\Delta u_{\perp} r_{b}=\varepsilon u_{0} / \gamma_{0} \simeq \varepsilon$. 
The peak on-axis brightness scales inversely quadratically with the electron beam physical emittance, $\varepsilon / \gamma_{0}$, linearly with the bunch charge, $q=e N_{e}$, and inversely with the electron bunch duration, $\Delta \tau$. Using the definition of the electron bunch brightness, $B_{e}=\gamma_{0}^{2} I_{b} / \varepsilon^{2}=$ $\gamma_{0}^{2} q / \varepsilon^{2} \Delta \tau$, we find that the $\mathrm{x}$-ray brightness is directly proportional to that quantity, thus emphasizing the importance of the electron beam quality for Compton scattering light sources.

While less critical, the laser plays an important role; in the linear regime, the laser pulse requirements for highbrightness X-ray operation can be summarized as follows: the spot size should be small, to maximize the incident photon density, and the pulse duration should be short compared to the Rayleigh length and the beta function of the electron beam in order to bring the overlap parameters $\eta$ and $\mu$ close to zero, as we have $\mathcal{O}(\eta, \mu) \leq \mathcal{O}(0,0)=$ $\sqrt{\pi}$. The laser fractional bandwidth, $\delta \omega$, however, should be commensurate with the electron beam relative energy spread, $\delta \gamma / 2$, and transverse momentum spread, $\Delta u_{\perp} / u_{0}$, to avoid significant degradation of the spectral density function, $S_{\varepsilon}\left(\chi, \delta \omega, \delta \gamma, \Delta u_{\perp}\right)$. In the nonlinear regime, described in the next section, these conditions are further constrained by the spectral broadening induced by the inhomogeneous laser ponderomotive force during the interaction.

\section{NONLINEAR SPECTRAL BROADENING}

Before evaluating this analytical expression of the peak on-axis brightness against our three-dimensional time and frequency-domain code, which has been benchmarked against experimental results, we study the spectral broadening induced by nonlinear effects for large values of the maximum laser normalized vector potential [7-9],

$$
A_{0}=2 \sqrt{\frac{N_{\lambda}}{\sqrt{\pi / 2}^{3} c \Delta t w_{0}^{2}} \lambda_{0} r_{0} \lambda_{C}}
$$

where $\lambda_{C}=r_{0} / \alpha$ is the electron Compton wavelength and $\alpha$ is the fine structure constant. Note that the local value of the normalized potential is simply given by

$$
A\left(x_{\mu}\right)=2 \sqrt{n_{\lambda}\left(x_{\mu}\right) \lambda_{0} r_{0} \lambda_{C}} .
$$

It is well known that the Doppler-upshifted radiation frequency of an electron subjected to an intense electromagnetic field must be corrected to account for the ponderomotive force of the incident laser pulse [7-10]; using our notation:

$$
\omega_{x}=\frac{\omega_{0}}{1+\left\langle A^{2}\right\rangle}\left(\frac{\gamma+u_{z}}{\gamma-u_{z}}\right)
$$

For a linearly polarized laser pulse,

$$
\left\langle A^{2}\right\rangle=\frac{1}{2}\left[2 \sqrt{n_{\lambda}\left(x_{\mu}\right) \lambda_{0} r_{0} \lambda_{C}}\right]^{2}=2 n_{\lambda}\left(x_{\mu}\right) \lambda_{0} r_{0} \lambda_{C} .
$$

This suggests that we can modify our derivation by per- forming the following replacement:

$$
\begin{aligned}
\delta\left(\omega_{x}-\omega \frac{\gamma+u_{z}}{\gamma-u_{z}}\right) & \rightarrow \delta\left[\omega_{x}-\frac{\omega}{1+2 n_{\lambda}\left(x_{\mu}\right) \lambda_{0} r_{0} \lambda_{C}}\right. \\
& \left.\times \frac{\gamma+u_{z}}{\gamma-u_{z}}\right] \\
= & \delta\left[\omega_{x}-\frac{\omega}{\rho\left(x_{\mu}\right)} \frac{\gamma+u_{z}}{\gamma-u_{z}}\right] .
\end{aligned}
$$

The physics behind this modification is that the frequency scattered by electrons is now a local variable, reflecting the local ponderomotive force experienced by the electrons. Proceeding along the same lines of the derivation presented in Sec. III, we then have

$$
\begin{aligned}
S_{\omega}= & \frac{r_{0}^{2}}{\sqrt{\pi} \Delta \omega}\left(\frac{\gamma+u}{\gamma-u}\right)\left(\frac{\gamma-u_{z}}{\gamma}\right) \rho\left(x_{\mu}\right) n_{\lambda}\left(x_{\mu}\right) n_{e}\left(x_{\mu}\right) \\
& \times \exp \left\{-\left[\frac{\omega_{x} \rho\left(x_{\mu}\right)\left[\left(\gamma-u_{z}\right) /\left(\gamma+u_{z}\right)\right]-\omega_{0}}{\Delta \omega}\right]^{2}\right\} \\
S_{\gamma} \simeq & \frac{2 r_{0}^{2} n_{e} n_{\lambda} \rho}{\sqrt{\pi} \omega_{0}} \frac{1+u_{\perp}^{2}}{\sqrt{\delta \omega^{2}+\delta \gamma^{2} \chi^{2} \rho^{2}\left(1+u_{\perp}^{2}\right)^{2}}} \\
& \times \exp \left\{-\frac{\left[\chi \rho\left(1+u_{\perp}^{2}\right)-1\right]^{2}}{\delta \omega^{2}+\delta \gamma^{2} \chi^{2} \rho^{2}\left(1+u_{\perp}^{2}\right)^{2}}\right\}
\end{aligned}
$$

and

$$
\begin{aligned}
S_{\varepsilon}\left(\chi, \delta \omega, \delta \gamma, \Delta u_{\perp}, x_{\mu}\right) \simeq & \frac{\sqrt{\pi}}{2 \chi \rho \Delta u_{\perp}^{2}} \exp \left\{\frac{\chi \rho-1}{2 \chi \rho \Delta u_{\perp}^{2}}\right. \\
& \left.\times\left[2+\frac{\delta \omega^{2}+\delta \gamma^{2} \chi^{2} \rho^{2}}{2 \chi \rho(\chi \rho-1) \Delta u_{\perp}^{2}}\right]\right\} \\
& \times 1-\Phi\left\{\frac{\chi \rho-1}{\sqrt{\delta \omega^{2}+\delta \gamma^{2} \chi^{2} \rho^{2}}}\right. \\
& \left.\times\left[1+\frac{\delta \omega^{2}+\delta \gamma^{2} \chi^{2} \rho^{2}}{2 \chi \rho(\chi \rho-1) \Delta u_{\perp}^{2}}\right]\right\} .
\end{aligned}
$$

To obtain the nonlinear brightness, we now need to perform the following integral:

$$
\begin{aligned}
\hat{B}_{x}= & \frac{8 \times 10^{-15} \gamma_{0}^{2} r_{0}^{2} c \chi}{\sqrt{\pi}} \int_{-\infty}^{+\infty} \rho\left(r=0, z, \frac{z}{c}\right) S_{\varepsilon} \\
& \times\left(\chi, \delta \omega, \delta \gamma, \Delta u_{\perp}, r=0, z, \frac{z}{c}\right) n_{e \lambda}\left(r=0, z, \frac{z}{c}\right) d z .
\end{aligned}
$$

Returning to the definition of the local ponderomotive force, and using previously defined variables, we have

$$
\begin{aligned}
\rho(r & \left.=0, z, \frac{z}{c}\right)=1+2 n_{\lambda}\left(r=0, z, \frac{z}{c}\right) \lambda_{0} r_{0} \lambda_{C} \\
& =1+\frac{A_{0}^{2}}{2} \frac{e^{-\bar{z}}}{1+\mu^{2} \bar{z}^{2}}=\rho(\bar{z}) .
\end{aligned}
$$


Finally, the explicit expression for the nonlinear brightness reads

$$
\begin{aligned}
\hat{B}_{x}= & \frac{4 \times 10^{-15}}{\pi^{3}} \frac{\gamma_{0}^{2}}{\varepsilon^{2}} \frac{N_{e} N_{\lambda}}{\Delta \tau} \frac{r_{0}^{2}}{w_{0}^{2}} \int_{-\infty}^{+\infty} \exp \frac{\chi \rho(\bar{z})-1}{2 \chi \rho(\bar{z}) \Delta u_{\perp}^{2}}\left\{2+\frac{\delta \omega^{2}+\delta \gamma^{2} \chi^{2} \rho^{2}(\bar{z})}{2 \chi \rho(\bar{z})[\chi \rho(\bar{z})-1] \Delta u_{\perp}^{2}}\right\} \\
& \left.\times\left(1-\Phi \frac{\chi \rho(\bar{z})-1}{\sqrt{\delta \omega^{2}+\delta \gamma^{2} \chi^{2} \rho^{2}(\bar{z})}}\left\{1+\frac{\delta \omega^{2}+\delta \gamma^{2} \chi^{2} \rho^{2}(\bar{z})}{2 \chi \rho(\bar{z})[\chi \rho(\bar{z})-1] \Delta u_{\perp}^{2}}\right\}\right]\right) \frac{e^{-\bar{z}^{2}} d \bar{z}}{\left(1+\eta^{2} \bar{z}^{2}\right)\left(1+\mu^{2} \bar{z}^{2}\right)}
\end{aligned}
$$

This expression is valid only for small values of $A_{0}^{2}$, in the weakly nonlinear regime, because it does not account for the radiation of harmonics $[6,7,9]$; however, it is sufficient to determine whether the laser intensity is small enough for the linear brightness scaling to apply; furthermore, it properly accounts for the downshift of the main spectral line due to ponderomotive force, as well as the inhomogeneous spectral broadening due to the variation of the ponderomotive force along the electron axis. It is sufficient, however, to determine the optimum laser pulse duration to maximize the source brightness, as discussed in Sec. VIB.

\section{LIMITATIONS OF THE ANALYTICAL THEORY}

In this section, a brief discussion is presented regarding the parameter space where the analytical theory applies. A number of assumptions are made in order to present an analytically tractable theory, which need to be reviewed in order to precisely define when the theory applies. First and foremost, the relative energy and transverse momentum spread of the electron beam, and the laser pulse bandwidth, are assumed to be small enough to warrant Taylorexpansions; typically, maintaining these below a few percent yields good agreement between the analytical brightness and full three-dimensional computer simulations. Second, the phase spaces of the laser pulse and electron bunch are uncorrelated: for example, frequency or energy chirping are not modeled here, as well as space-charge effects and coherent synchrotron radiation along the electron beam line upstream of the final focus. Other limitations include very small focal radii, where the laser pulse wave-front curvature becomes important, along with the electron beam divergence; these effects appear for values below a few $\mu \mathrm{m}$. Finally, as mentioned earlier, the incident laser pulse is modeled in terms of photons, as opposed to a classical coherent electromagnetic field, and the electrons are described via the Compton scattering differential cross section, to obtain a description of the scattered radiation both in the time and frequency domains. The photon description, however, imposes limitations to the modeling of nonlinear effects, where the ponderomotive force plays an important role in modulating the axial dynamics of the particles. In particular, oscillations in the spectral fluence that have been described in Refs. [18,19], are more difficult to describe within the photon model, as they require one to take into account the instantaneous state of motion of the electron at the time of scattering. Finally, we note that we have considered emittance-dominated beams, which is generally a good assumption for most current and planned Compton scattering light sources.

\section{COMPARISON BETWEEN ANALYTICAL BRIGHTNESS AND THREE-DIMENSIONAL CODES}

Having derived an analytical expression for the peak onaxis brightness and prescribed a simple analysis of weakly nonlinear effects, we now focus on a detailed comparison between those results and our three-dimensional codes $[8,12]$, which have been thoroughly benchmarked against ongoing Compton scattering experiments at LLNL [1113]. For example, Fig. 5 shows the excellent agreement

\section{Calculated Profile}
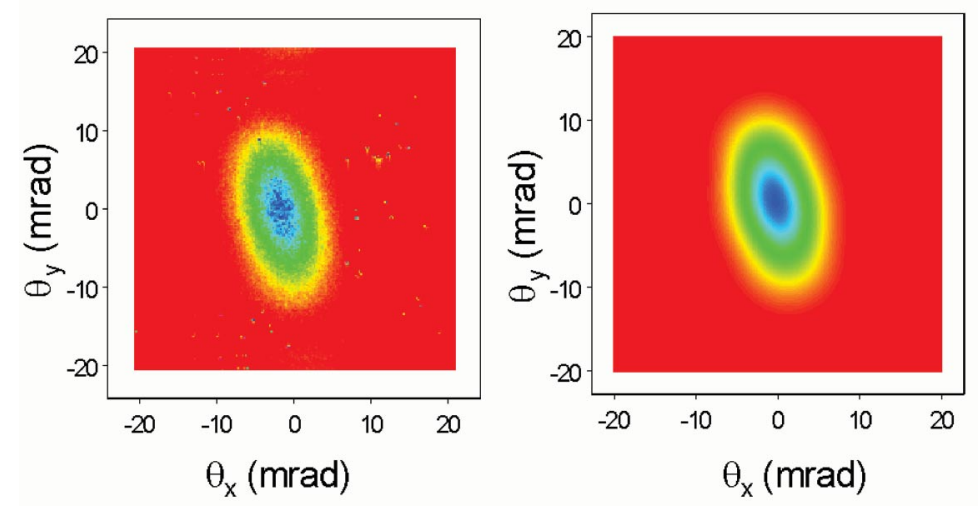

FIG. 5. (Color) Comparison between the experimentally measured and computationally predicted x-ray angular energy distribution at the PLEIADES facility. The maximum photon energy produced as of this writing is $140 \mathrm{keV}$. 
obtained between the three-dimensional codes and the experimentally measured angular x-ray energy distribution. The vertical axes are color coded linearly, with the same scale, and the integrated x-ray dose agree to within $10 \%$. This deviation is attributed to the accuracy with which the various moments of the electron and laser phase spaces can be measured, including energy, energy distribution, emittance, focal spot size, beta function, Rayleigh range, and charge. For more detail, we refer the reader to references [11-13].

\section{A. Laser and linac parameters, $x$-ray brightness}

The specific parameters used in these simulations correspond to an optimized S-band linac using a 1.6-cell rf gun with symmetrized racetrack cavities operating with a peak photocathode field of $140 \mathrm{MeV} / \mathrm{m}$, a fully spatially and temporally shaped UV photocathode laser pulse [20], and full implementation of emittance compensation. For such a system, a normalized rms emittance of $<1 \mathrm{~mm}$. mrad is expected at a charge of $1 \mathrm{nC}$. This is confirmed by PARMELA simulations, showing that $0.6 \mathrm{~mm} \cdot \mathrm{mrad}$ is possible at a full $\mathrm{nC}$ of charge, as shown in Fig. 6, for a $250 \mathrm{MeV}$ beam. Five 2.5-meter long rf sections operating at a gradient of $20 \mathrm{MeV} / \mathrm{m}$ to minimize dark current, and using symmetrized rf feeds to minimize dipole and multipole field aberrations, then accelerate the bunch. One of the critical parameters in the $\mathrm{x}$-ray brightness scaling is the electron bunch duration, $\Delta \tau$ : as shown in Fig. 4, the x-ray flash has essentially the same temporal length as the electron beam. Therefore, it is highly desirable to produce very short electron bunches to generate ultrashort x-ray flashes. The five-section linac architecture allows for the implementation of both velocity and chicane compression schemes, which have already demonstrated electron bunch lengths as short as $300 \mathrm{fs} r \mathrm{rs}$ and $80 \mathrm{fs}$ rms at PLEIADES [11-13] and at the Sub-Picosecond Pulse Source [21,22], respectively. However, some care should be taken when performing pulse compression, in order to minimize coherent synchrotron radiation, space charge, and nonthermal emittance growth.

The main drive laser specifications are as follows: pulse energy in the $1-5 \mathrm{~J}$ range, duration of $5 \mathrm{ps}$ FTL, $10-100 \mathrm{~Hz}$ repetition rate, and diode pumping for stability. Adaptive optics could be used to provide for a high-quality laser focal region. In the simulations presented here, we have consistently used $1 \mathrm{~J}$ of laser energy.

Optimized parameters used in the following simulation are summarized in Table I. For these parameters, the Rayleigh length is $z_{0}=1.257 \mathrm{~mm}$, the normalized vector potential is $A_{0}^{2}=0.019$, and the beta function is $\beta_{f}=$ $49 \mathrm{~mm}$. The peak on-axis brightness is shown in Fig. 7, where the squares are produced by a fully threedimensional time and frequency-domain code [12], while the solid line corresponds to the linear analytical model derived in Sec. III; the agreement is very good, and the
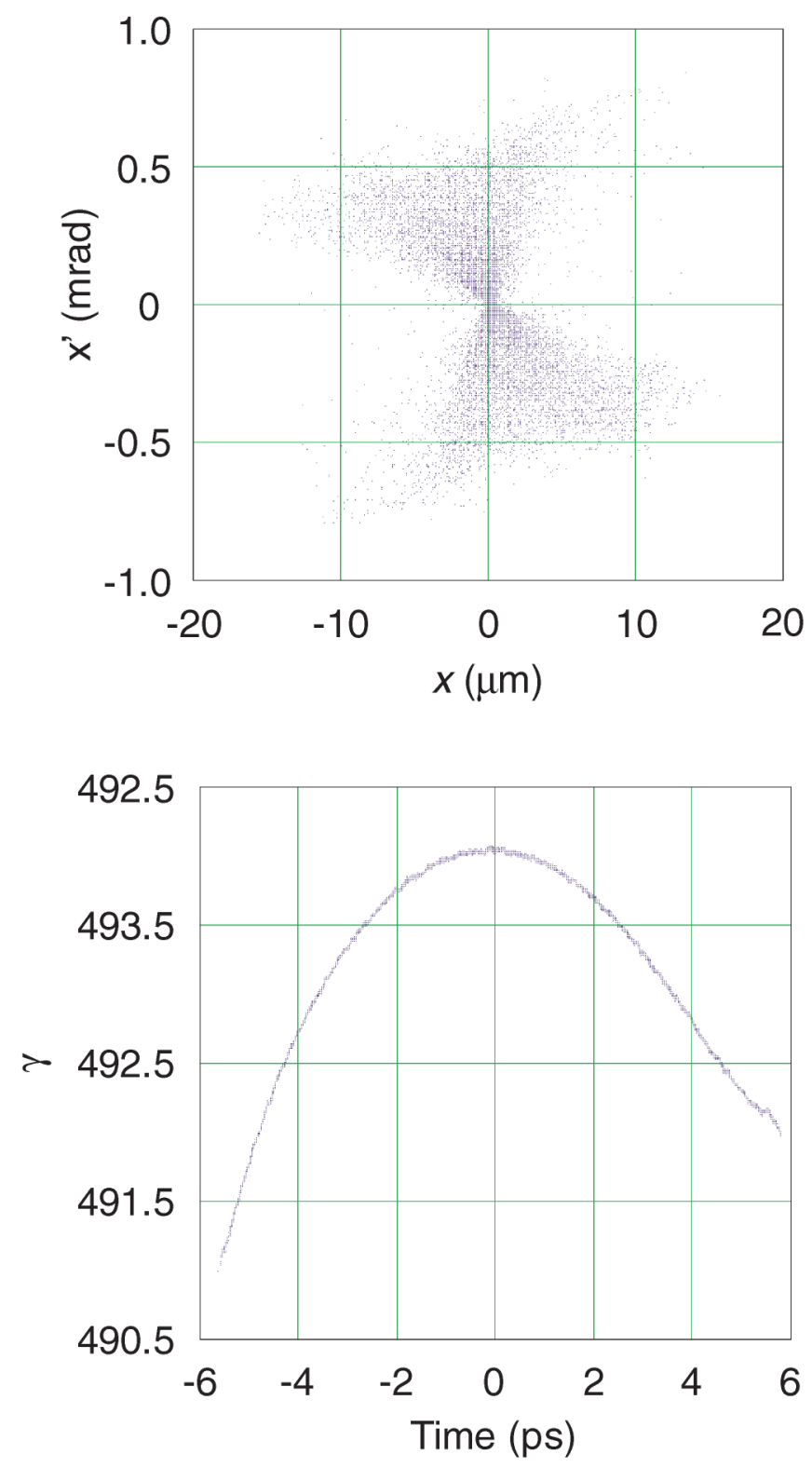

FIG. 6. (Color) PARMELA simulations for a $1 \mathrm{nC}, 0.6 \mathrm{~mm} \cdot \mathrm{mrad}$, $250 \mathrm{MeV}$ beam (see text).

variations seen on the code results are caused by statistical noise, as this first example was simulated with 20000 macroparticles. Indeed, for 200000 macroparticles, the agreement is even better, as seen in Fig. 8. In particular, the maximum value of the peak on-axis brightness predicted by the code is $3.736 \times 10^{21}$, in synchrotron units, while the analytical model yields $3.677 \times 10^{21}$, with a relative indetermination of $1.6 \%$. To verify the close match between the electron beam phase space input to the threedimensional code and the Gaussian distributions used in the analytical theory, histograms are compared with the latter.

Next, the electron beam energy is raised to $250 \mathrm{MeV}$, while all other parameters are kept constant. To minimize statistical noise, as explained above, we run 200000 par- 
TABLE I. Linac and laser parameters.

\begin{tabular}{lcc}
\hline \hline & \multicolumn{1}{c}{ Linac } & Laser \\
\hline Particle energy & $\gamma_{0} m_{0} c^{2} / e=50 \mathrm{MeV}$ & $h c / e \lambda_{0}=1.2398 \mathrm{eV}$ \\
Number of particles & $q=N_{e} e=1 \mathrm{nC}$ & $W=N_{\lambda} \omega_{0}=1 \mathrm{~J}$ \\
Pulse duration & $\Delta \tau / \sqrt{2}=100 \mathrm{fs} \mathrm{rms}$ & $\Delta t=5 \mathrm{ps}\left(\right.$ intensity $\left.1 / e^{2}\right)$ \\
Focal spot radius & $r_{b} / \sqrt{2}=10 \mu \mathrm{m} \mathrm{rms}$ & $\left.w=20 \mu \mathrm{m} \mathrm{(intensity} 1 / e^{2}\right)$ \\
Energy spread & $\Delta \gamma / \sqrt{2} \gamma_{0}=0.1 \% \mathrm{rms}$ & $\Delta \omega=\sqrt{2} / \Delta t(\mathrm{FTL})$ \\
Transverse phase space & $\varepsilon / 2=1 \mathrm{~mm} \cdot \mathrm{mrad} \mathrm{rms}$ & $\Delta k_{\perp} / k_{0} 1$ \\
\hline \hline
\end{tabular}

ticles. The result is shown in Fig. 9. Again, excellent agreement is obtained between the three-dimensional time and frequency-domain code and the analytical brightness, with a maximum of $9.198 \times 10^{22}$, and $9.04 \times 10^{22}$ synchrotron units, respectively, at a photon energy of $1.19 \mathrm{MeV}$. Note that the peak brightness has effectively scaled as $\gamma_{0}^{2}$, since the brightness at $250 \mathrm{MeV}$ is very nearly 25 times higher than that obtained at $50 \mathrm{MeV}$. Clearly, this would represent an exceptionally bright source, many orders of magnitude above the output of the highest energy synchrotron currently in operation, although the gain in average brightness is less spectacular, as the practical repetition rate of such a source would probably be in the $10-100 \mathrm{~Hz}$ range. We also note that reaching $1 \mathrm{kHz}$ repetition rate with $1 \mathrm{~J}$ per pulse, corresponding to $1 \mathrm{~kW}$ average power, may soon be possible by using high average power fiber laser technology, currently under development [23], and hyperdispersion compressors. To further establish the $\gamma^{2}$ scaling of the peak brightness, the codes have been run at $100,150,200$, and $250 \mathrm{MeV}$, both for a fixed energy spread of $\Delta \gamma=0.05 \mathrm{MeV}$ and for a fixed relative energy spread of $\Delta \gamma / \gamma_{0}=0.1 \%$; the result, shown in Fig. 10 clearly confirm that scaling.

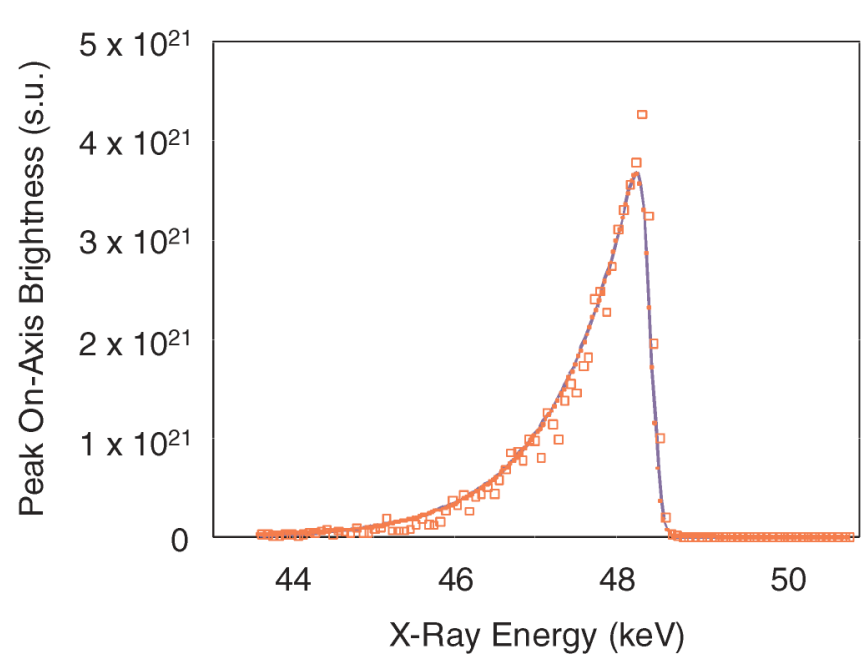

FIG. 7. (Color) Peak spectral on-axis brightness as a function of photon energy for the electron bunch and drive laser pulse parameters presented in Table I. The blue curve is the analytical brightness, while the red squares correspond to threedimensional computer simulations using 20000 macroparticles.
Another important aspect of the x-ray phase space is the fact that, to lowest order, the brightness is nearly independent from the electron beam spot size: one trades divergence and spot size, in exact parallel with the electron

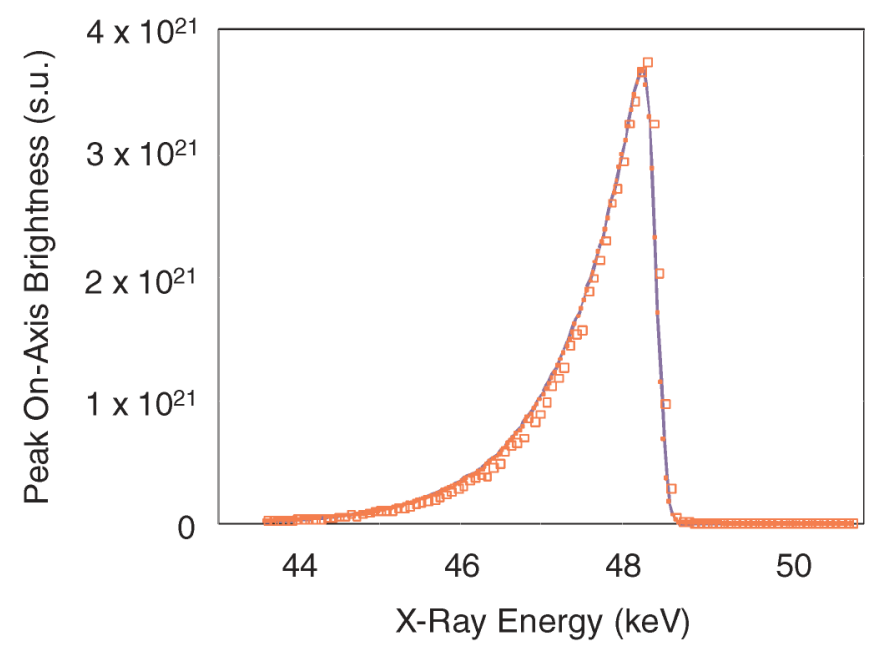

FIG. 8. (Color) Identical to Fig. 7, with 200000 macroparticles.

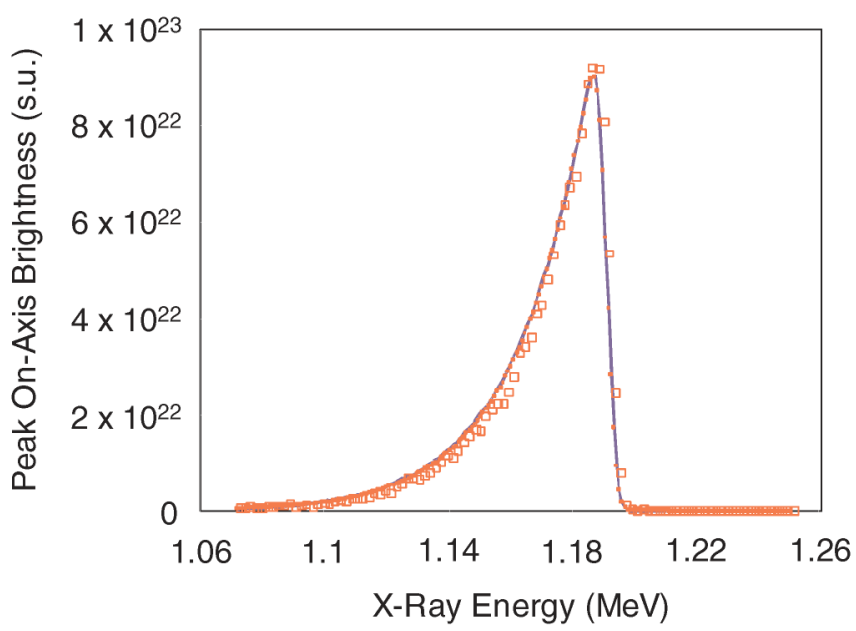

FIG. 9. (Color) Peak spectral on-axis brightness as a function of photon energy for the electron bunch and drive laser pulse parameters presented in Table I, except for the beam energy, which is now $250 \mathrm{MeV}$. The blue curve is the analytical brightness, while the red squares correspond to three-dimensional computer simulations using 200000 macroparticles. 


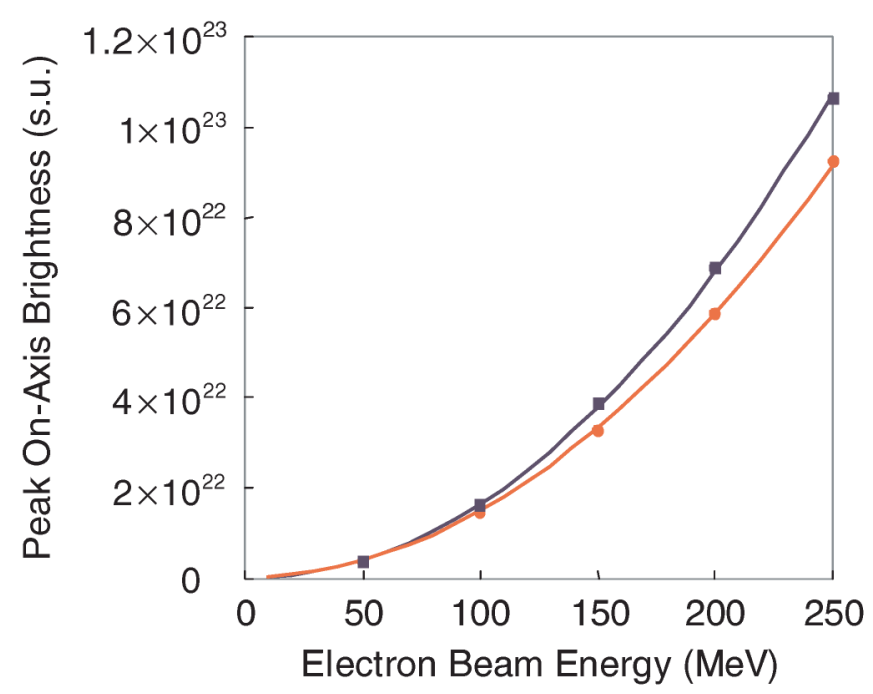

FIG. 10. (Color) Spectral maximum of the peak on-axis brightness as a function of the electron beam energy. The solid blue curve corresponds to the analytical brightness for a fixed energy spread of $0.05 \mathrm{MeV}$, while the solid red curve is generated using the analytical brightness for a fixed relative energy spread of $0.1 \%$. The blue squares and red circles correspond to threedimensional computer simulations using 200000 macroparticles.

beam phase space. This does not hold exactly, however, because the overlap between the electron and laser beams varies with the electron beam spot size. Furthermore, the spectral content of the $\mathrm{x}$ rays is also influenced by the electron beam divergence; the combination of these two effects, namely, overlap and emittance-induced spectral broadening explains the slow variation of the x-ray brightness with the electron beam radius. More specifically, it is important to note that the x-ray spot size is not necessarily strongly dependent on the electron beam spot size. The fact that the brightness is primarily a function of the electron beam normalized emittance, and not the spot size, is derived from the combination of the dependence of the x-ray dose on both the electron beam and laser beam spot sizes, through the overlap function, and the dependence of the $\mathrm{x}$ ray divergence and spectrum on the electron beam divergence in the limit where the electron beam divergence, or emittance, is the dominant factor in determining the $\mathrm{x}$-ray spectrum. The variation from the simple scaling is due primarily to the two following facts: when the electron spot size is big, the spectral width will be dominated by the electron beam energy spread and the laser bandwidth, hence the brightness should be lower than suggested by the scaling; when the electron spot is small, the beta function will become shorter than the possible interaction length, hence, degrade the overlap function, and the brightness. Additionally, the large transverse velocity can also lead to higher order terms leading to additional broadening of the spectrum.

This can be studied systematically, as shown in Fig. 11, where the behavior of the peak on-axis spectral brightness
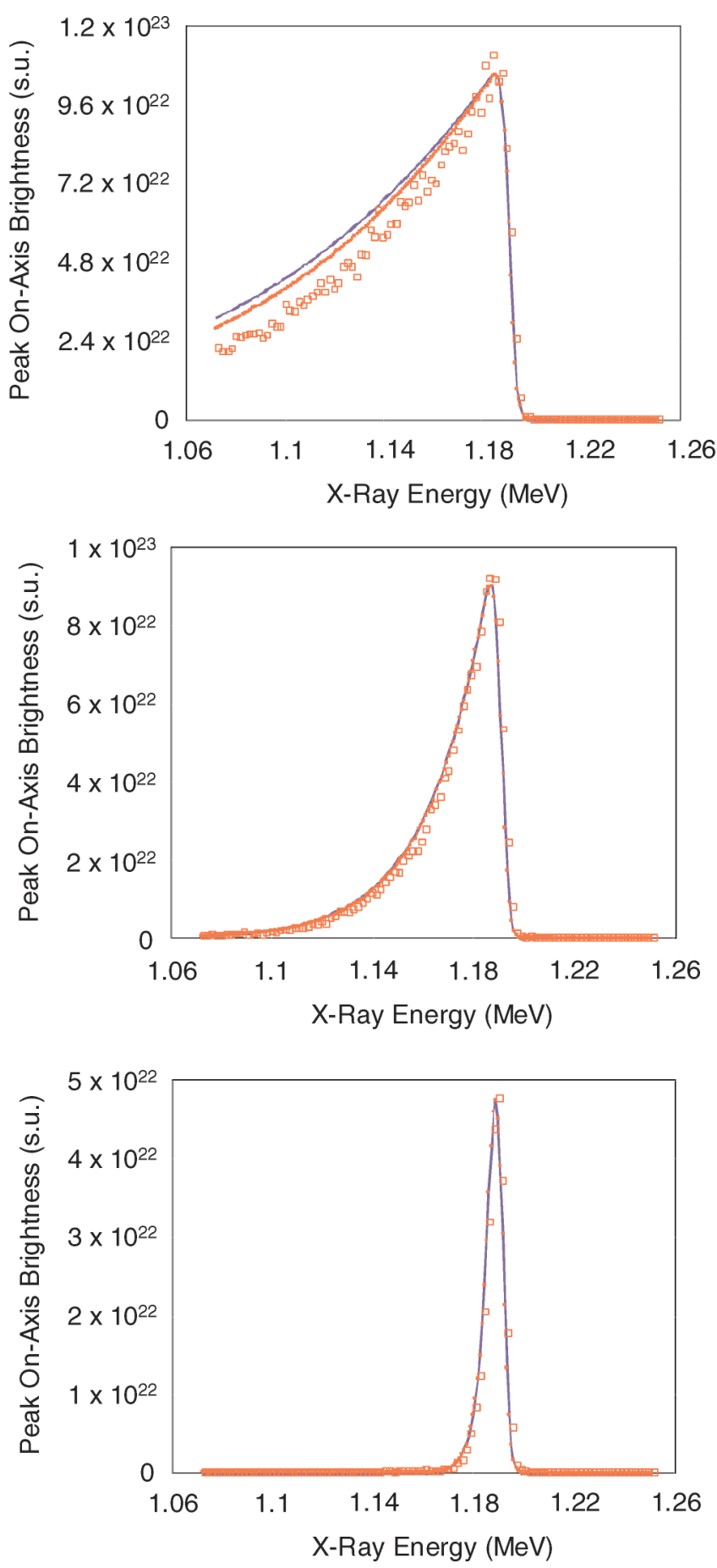

FIG. 11. (Color) Top: peak spectral on-axis brightness as a function of photon energy for an electron beam focal radius $r_{b} / \sqrt{2}=5 \mu \mathrm{m}$ rms. Center: peak spectral on-axis brightness as a function of photon energy for an electron beam focal radius $r_{b} / \sqrt{2}=10 \mu \mathrm{m}$ rms. Bottom: peak spectral on-axis brightness as a function of photon energy for an electron beam focal radius $r_{b} / \sqrt{2}=25 \mu \mathrm{m} \mathrm{rms}$. The electron beam energy is $250 \mathrm{MeV}$; all other parameters are given in Table I. In the top figure, the blue curve corresponds to Eq. (5), while the red one is obtained from a direct computer integration of the overlap integral; a slight deviation is only observed at very small radii. The red squares are generated by three-dimensional computer simulations. 
is shown for 3 different values of the electron beam focal spot size: 5,10 , and $25 \mu \mathrm{m}$. The spectral broadening due to the large electron beam divergence for small spot sizes is clearly visible; for large focal beam radii, the spectrum is dominated by the laser bandwidth and the relative energy spread of the electron beam. The maximum value of the peak on-axis brightness can then be plotted as a function of the electron beam focal radius, as shown in Fig. 12: instead of scaling rapidly like the inverse source size and electron beam density, $1 / r_{b}^{4}$, the variation of the brightness is slow, and depends mostly on the aforementioned combination of divergence-induced spectral broadening and beams overlap integral. For applications requiring narrow x-ray bandwidth, one can use a relatively large spot size, while the brightness can be maximized for small electron beam focal spots, at the expense of spectral purity. The discrepancy between the three-dimensional code and the analytical theory for very small values of $r_{b}$ corresponds to the fact that the assumption of small transverse velocity spread, $\Delta u_{\perp} / u_{0}$, begins to break down for these extremely small electron beam focal spot sizes.

\section{B. Optimum laser pulse duration and bandwidth}

In the case of Fourier-transform-limited laser pulses, the relation between the pulse duration, $\Delta t$, and the spectral bandwidth, $\Delta \omega$, as defined in the previous sections is $\Delta \omega \Delta t=\sqrt{2}$. For large values of the laser pulse duration, the laser bandwidth is very narrow, and the normalized vector potential is small, which allows for minimal linear

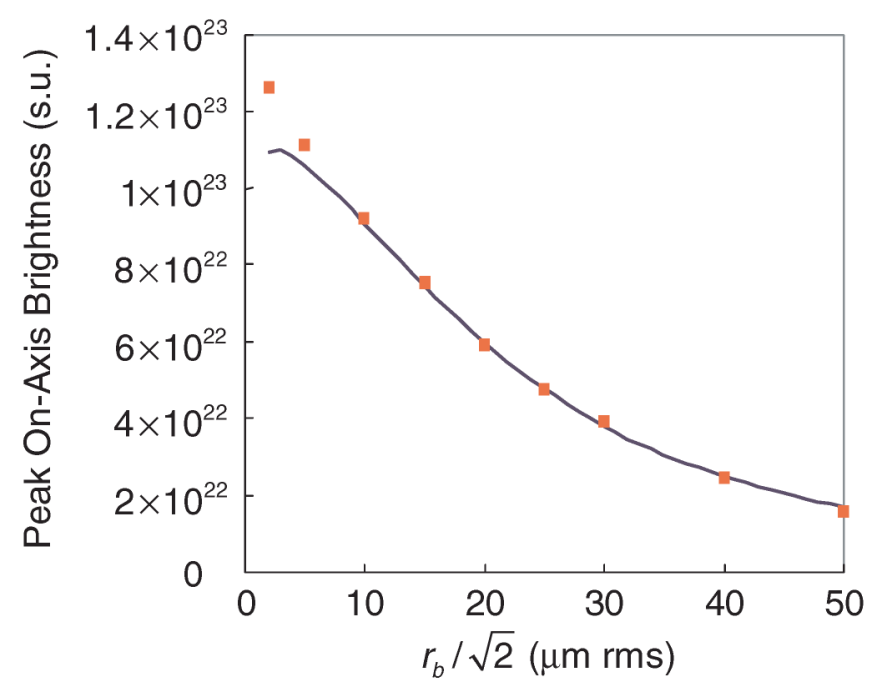

FIG. 12. (Color) Spectral maximum of the peak on-axis brightness as a function of the electron beam focal radius. The solid blue curve is generated using the analytical brightness while the red squares correspond to three-dimensional computer simulations using 200000 macroparticles. The deviation observed at small radii corresponds to the fact that the assumption of small transverse velocity spread, $\Delta u_{\perp} / u_{0}$, begins to break down for these extremely small electron beam focal spot sizes. and nonlinear x-ray spectral broadening; however, the overlap integral becomes small as the normalized parameters $\eta=k_{f} c \Delta t / 2 \sqrt{2}$ and $\mu=c \Delta t / 2 \sqrt{2} z_{0}$ become large. Conversely, for ultrashort laser pulses, the fractional laser bandwidth contributes strongly to the x-ray spectral bandwidth, and nonlinear effects become important, further degrading the x-ray brightness. To quantify these effects, the peak on-axis spectral brightness is derived as a function of the drive laser pulse duration, while all other parameters, including the laser pulse energy and spot size, are fixed and described in Table I, except the electron beam energy, which is $250 \mathrm{MeV}$; the results are shown in Figs. 13 and 14. When nonlinear effects are neglected, a relatively large range of laser pulse durations yields high-brightness operation; the limits are set by the laser bandwidth for short pulses, which broadens the x-ray spectrum, and by diffraction for long pulses. Accounting for nonlinear effects, however, reveals a much tighter constraint on the drive laser pulse duration, as shown in Fig. 14, where the strength of the ponderomotive force is also indicated: the optimum lies in the $2-10 \mathrm{ps}$, range and a duration of $5 \mathrm{ps}$ FTL represents a good design point. This is an important result, as it guides the choice of technology regarding the drive laser, as outlined in Sec. VI A.

The various sources of spectral broadening for the scattered $\mathrm{x}$ rays are summarized in Table II; they also help define the transition from Thomson, or recoilless scattering to Compton scattering, as explained below.

While it is difficult to define the transition from Thomson to Compton scattering because there is no scale for the recoil, a pragmatic approach consists in comparing the various contributions to spectral broadening listed above: if the relative recoil term is small compared to the scattered x-ray fractional bandwidth, one operates in the Thomson scattering limit, while when recoil contributes

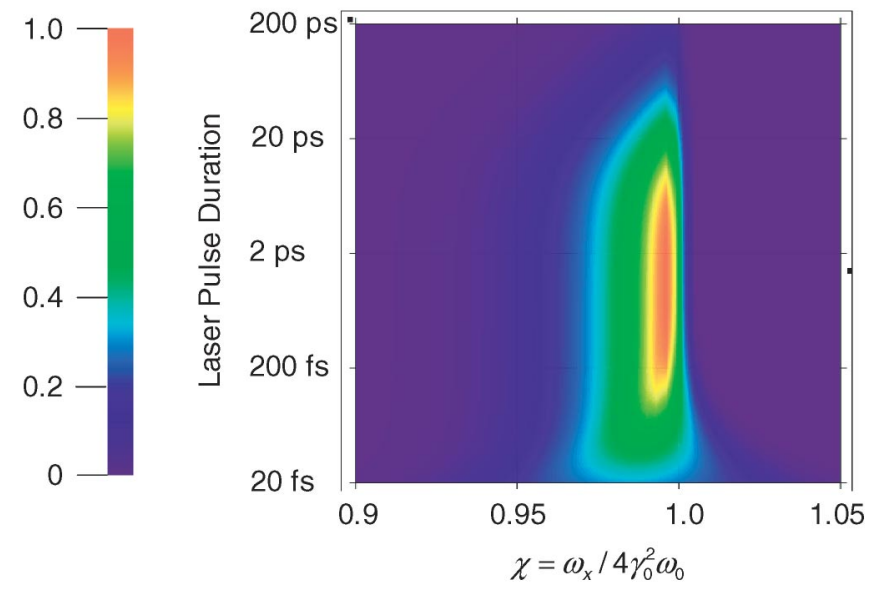

FIG. 13. (Color) Peak spectral on-axis brightness as a function of the normalized Doppler-shifted frequency, $\chi=\omega_{x} / 4 \gamma_{0}^{2} \omega_{0}$, and laser pulse duration, $\Delta t$. All other parameters are fixed, as described in the text, and nonlinear effects are ignored. The color scale is linear, with arbitrary units. 

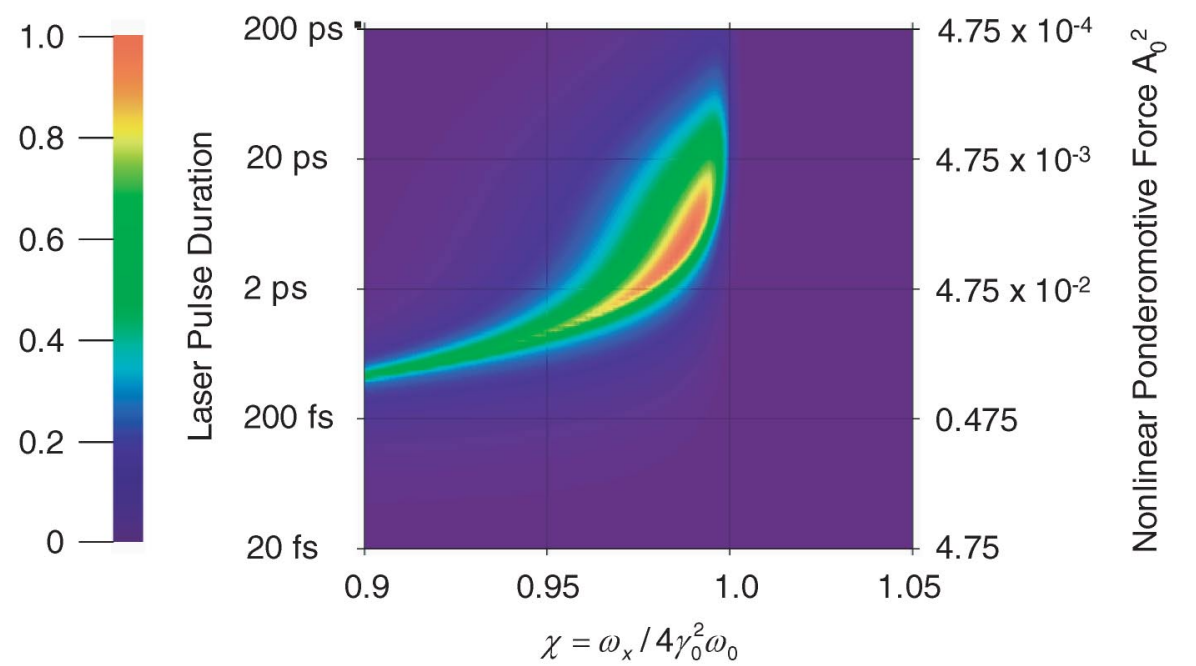

FIG. 14. (Color) Identical to Fig. 13, but nonlinear effects are included. The color scale is linear, with arbitrary units.

significantly to the $\mathrm{x}$-ray spectral width, Compton scattering best describes the process.

\section{Comparison with correlated electron beam phase space}

The Gaussian distributions used to obtain an analytical expression of the brightness do not contain the type of correlations that one typically observes in real electron beams; in order to further evaluate the robustness of our theoretical model, we have performed a detailed comparison between the analytical theory and PARMELA simulations interfaced to the aforementioned three-dimensional time and frequency-domain x-ray code for a specific example. The electron beam phase space is that given in Fig. 6, and corresponds to the system outlined in Sec. VI A; focusing is provided by a quadrupole triplet, which produces a tight focal spot shown in Fig. 15. The corresponding three-dimensional electron distribution at focus is shown in Fig. 16, for 10000 macroparticles. To see how the correlated phase space given in this example deviates from the idealized Gaussian distributions used in the analytical model, histograms are presented in Figs. 17 and 18, and compared with simple distributions: for example, the radial particle distribution shown in Fig. 17 (top) is well

TABLE II. Various sources of spectral broadening for scattered $\mathrm{x}$ rays.

\begin{tabular}{lc}
\hline \hline Spectral broadening parameter & Physical origin \\
\hline$\Delta \omega / \omega_{0}$ & Laser fractional bandwidth \\
$c \Delta k_{\perp} / \omega_{0}$ & Laser focusing \\
$\Delta \gamma / \gamma_{0}$ & Electron beam energy spread \\
$\varepsilon / \gamma_{0} r_{b}$ & Electron beam focusing \\
$A_{0}^{2}$ & Nonlinear ponderomotive force \\
$2 \gamma_{0} \omega_{0} / m_{0} c^{2}$ & Compton scattering recoil \\
\hline \hline
\end{tabular}

approximated by a function of the type $\sqrt{r / r_{b}} e^{-r / r_{b}}$, whereas the Gaussian equivalent takes the form $r e^{-r^{2} / r_{b}^{2}} / r_{b}$; the temporal distribution, on the other hand, closely resembles a super-Gaussian of the form $\exp \left[-(t / \Delta \tau)^{8}\right]$, as shown in Fig. 17 (bottom). The distribution that most deviates from a Gaussian, however, is that of the particle energy, shown in Fig. 18 (top), as it is strictly bounded on the high side by the maximum linac accelerating gradient, obtained at the optimum phase. Finally, the distribution of transverse momentum, shown in Fig. 18 (bottom), substantially deviates from the $u_{\perp} \exp \left[-\left(u_{\perp} / \Delta u_{\perp}\right)^{2}\right] / \Delta u_{\perp}$ distribution used in the model. Nonetheless, the analytical brightness still com-

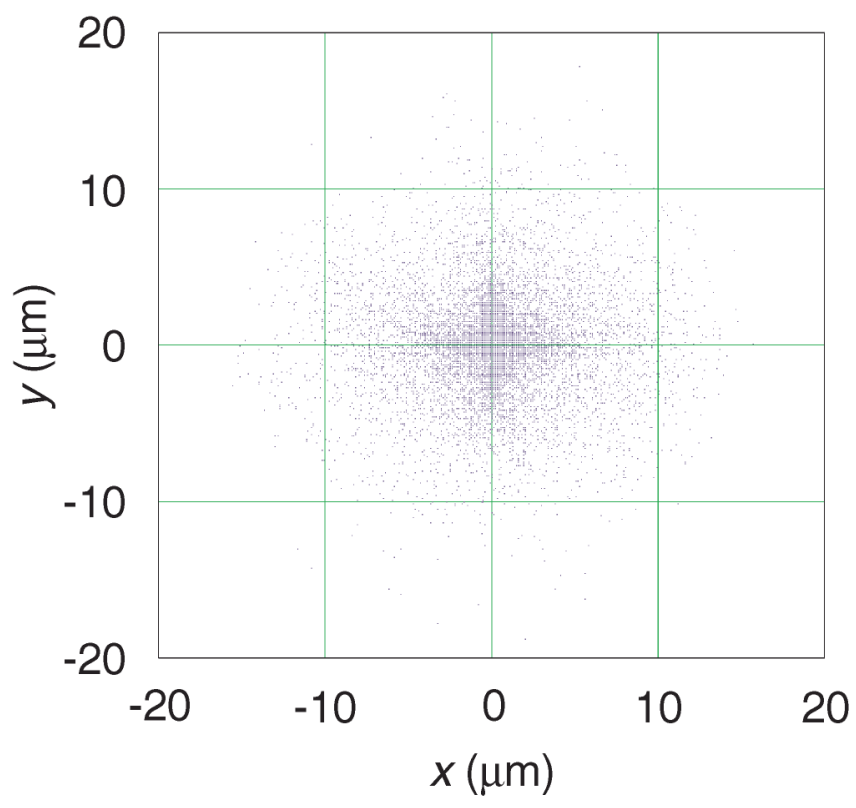

FIG. 15. (Color) Electron beam focal distribution generated with PARMELA. 


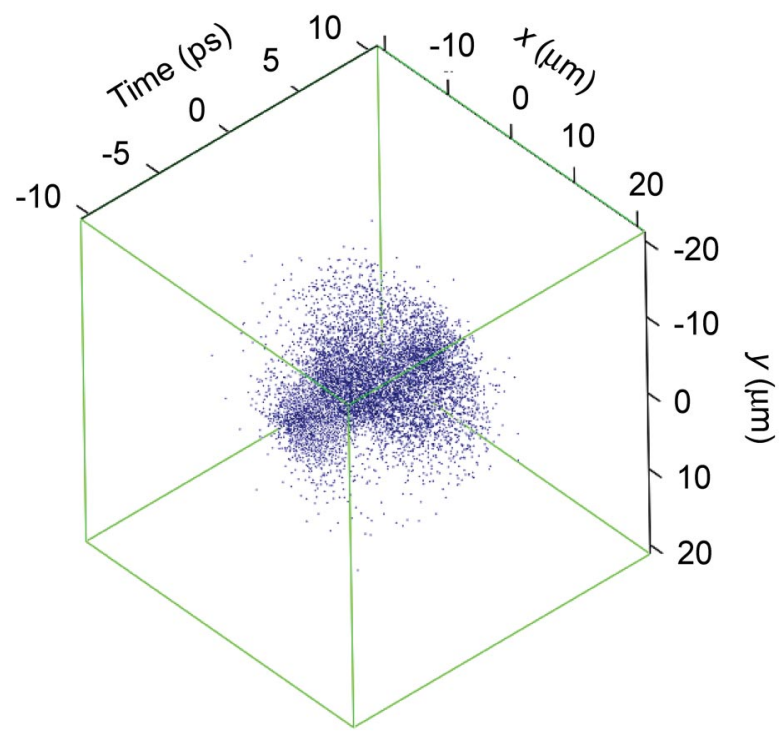

FIG. 16. (Color) Three-dimensional electron distribution at focus, generated with PARMELA using 10000 macroparticles.
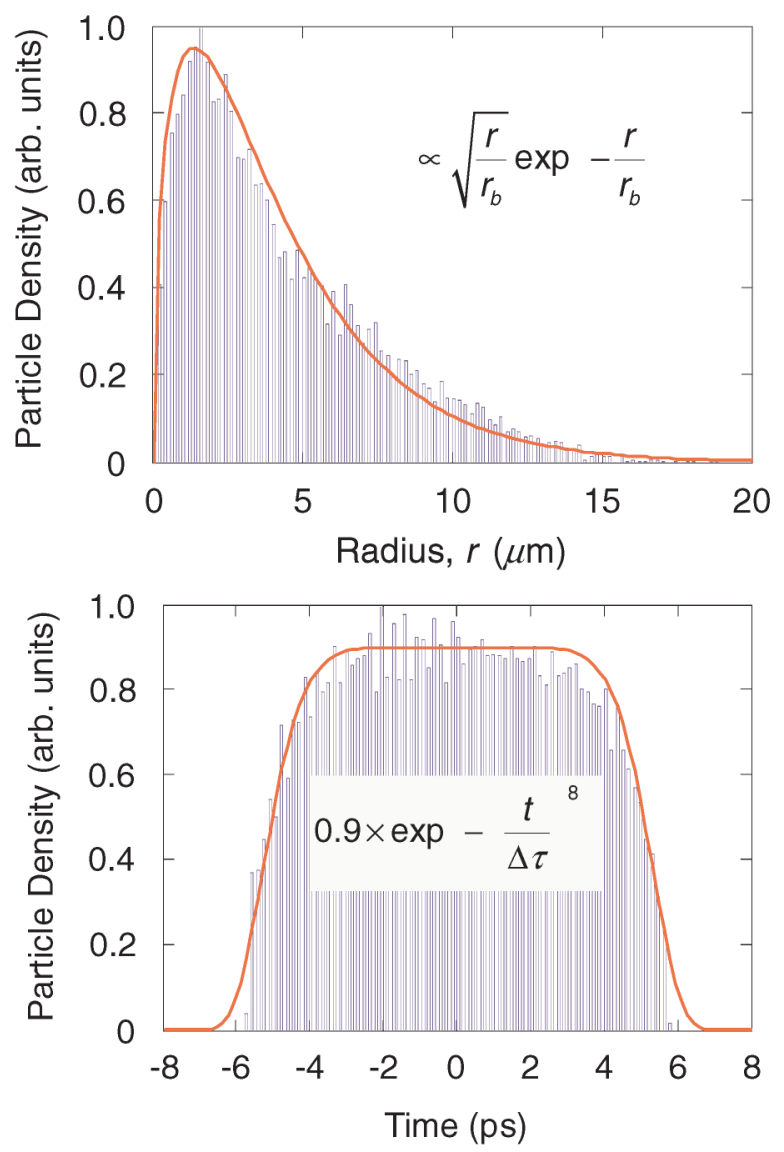

FIG. 17. (Color) Top: histogram of the radial particle distribution at focus; the red curve shows that the particle density behaves as $\sqrt{r / r_{b}} e^{-r / r_{b}}$. Bottom: temporal histogram of the electron bunch; the red curve is a super-Gaussian, of the form $\exp \left[-(t / \Delta \tau)^{8}\right]$.
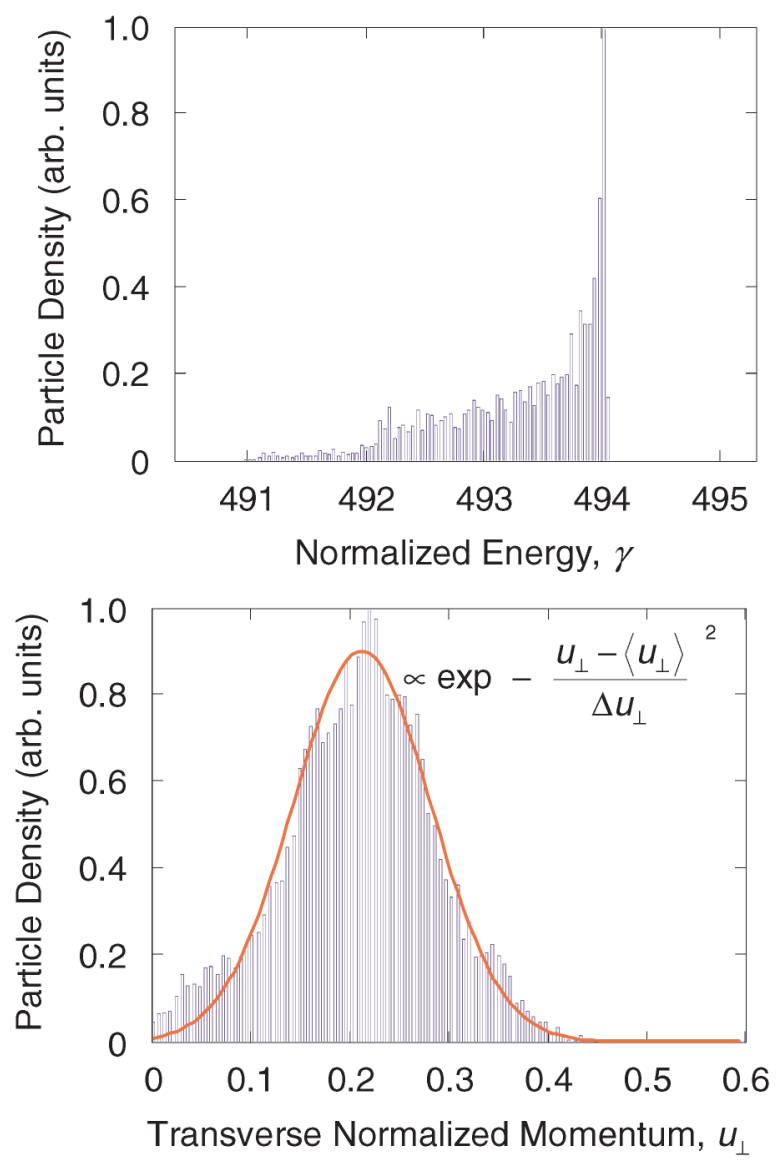

FIG. 18. (Color) Top: histogram of the particle energy. Bottom: histogram of the particle transverse momentum; the red curve is a Gaussian fit.

pares well with the result of a full three-dimensional simulation, as shown in Fig. 19. Here, we have used the PARMELA phase space to calculate the average value and rms deviation of the parameters used in the theory, and made small adjustments to optimize the fit: $\langle\gamma\rangle=493.33$, $\Delta \gamma=\sqrt{2} \Delta \gamma_{\mathrm{rms}}=0.954, \quad \Delta \tau=\sqrt{2} \Delta \tau_{\mathrm{rms}}=4.208 \mathrm{ps}$, $r_{b}=1.13 r_{b \mathrm{rms}}=6.23 \mu \mathrm{m}$, and $\Delta u_{\perp}=\Delta u_{\perp \mathrm{rms}}=0.225$. The main discrepancy is mainly due to the non-Gaussian nature of the energy distribution shown in Fig. 18 (top). We also note that the peak brightness presented here for a full PARMELA simulation of the electron beam and a full threedimensional x-ray simulation, with an uncompressed electron beam, is quite high and reaches $8 \times 10^{21}$ in synchrotron units; an electron bunch compression factor of 15 , down to $200 \mathrm{fs}$ rms would generate a brightness of $1.2 \times$ $10^{23}$ synchrotron units, provided the emittance is not degraded in the process. These numbers confirm the feasibility of the results presented in Sec. VI A.

\section{CONCLUSIONS}

High-energy, high-brightness Compton scattering light sources offer a promising development path toward 


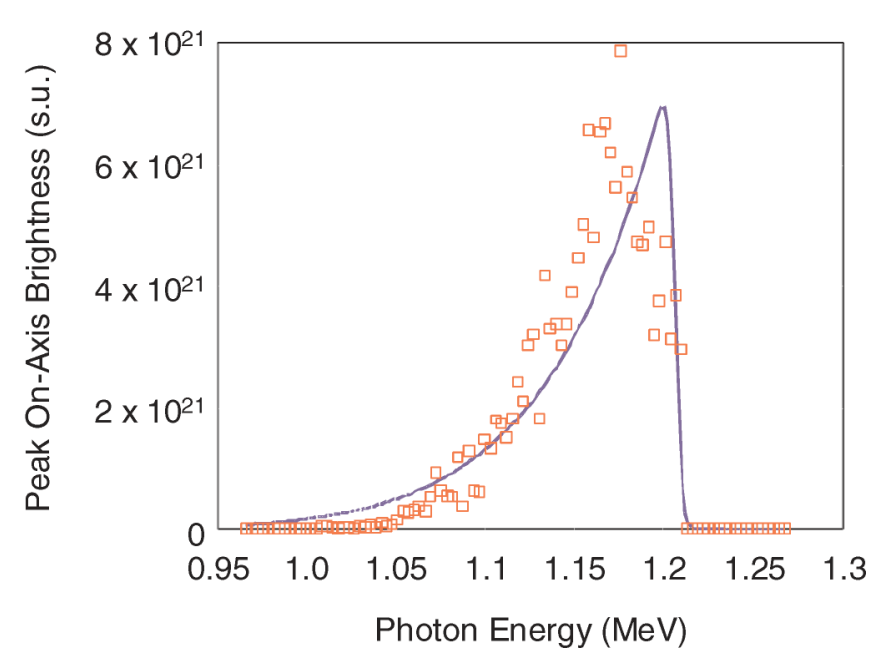

FIG. 19. (Color) Peak spectral on-axis brightness as a function of photon energy for the $250 \mathrm{MeV}$ electron bunch simulated by PARMELA. The blue curve is the analytical brightness, while the red squares correspond to three-dimensional computer simulations using 10000 macroparticles.

an unexplored region of the brightness-energy chart, with a projected peak on-axis brightness in the $10^{20}-10^{25}$ photons $/ \mathrm{mm}^{2} \times \mathrm{mrad}^{2} \times \mathrm{s} \times 0.1 \%$ bandwidth range, and photon energies between $100 \mathrm{keV}$ and $10 \mathrm{MeV}$. Such novel light sources are made possible by the combination of new technologies, including highbrightness, high-gradient symmetrized rf guns, spatial and temporal laser pulse shaping, diode-pumped solidstate lasers, and optical parametric amplification. A wide array of important applications would greatly benefit from such new hard $\mathrm{x}$-ray and $\gamma$-ray sources, including nuclear fluorescence spectroscopy, time-resolved positron annihilation spectroscopy, and $\mathrm{MeV}$ flash radiography. The brightness scaling of Compton scattering light sources has been derived and compared to fully three-dimensional codes that have been extensively benchmarked against experiments performed at LLNL; the agreement is found to be excellent over a wide parameter range, and the analytical brightness expression allows one to quickly explore the wide parameter space comprising both the electron beam and the laser pulse phase spaces to optimize the design of a compact, tunable, high-brightness light source using a Joule-class, 5 ps FTL laser and a low normalized emittance $(1 \mathrm{~mm} \cdot \mathrm{mrad}, 1 \mathrm{nC})$, relativistic $(50-250 \mathrm{MeV})$ electron beam. As a caveat, we note that in the case of a correlated phase space, with non-Gaussian distributions, such as those often produced by realistic PARMELA simulations, the analytical theory does deviate slightly from the full three-dimensional code results, but is still effective in helping predict the peak brightness and spectral character- istics of the source. Finally, nonlinear effects have also been included, and shown to play an important role in optimizing the drive laser pulse duration.

\section{ACKNOWLEDGMENTS}

This work was performed under the auspices of the U.S. Department of Energy by University of California, Lawrence Livermore National Laboratory under Contract No. W-7405-Eng-48. One of us (F. V. H.) would also like to acknowledge very useful discussions with D.T. Santa Maria.

[1] J. Arthur, G. Materlik, R. Tatchyn, and H. Winick, Rev. Sci. Instrum. 66, 1987 (1995).

[2] http://www-ssrl.slac.stanford.edu/lcls/cdr/

[3] F. Ciocci, G. Dattoli, A. Torre, and A. Renieri, Insertion Devices for Synchrotron Radiation and Free-Electron Lasers (World Scientific, Singapore, 2000).

[4] N. Pietralla et al., Phys. Rev. Lett. 88, 012502 (2002).

[5] C. Bemporad, R. H. Milburn, N. Tanaka, and M. Fotino, Phys. Rev. 138, B1546 (1965).

[6] E. Esarey, S. K. Ride, and P. Sprangle, Phys. Rev. E 48, 3003 (1993).

[7] F. V. Hartemann and A. K. Kerman, Phys. Rev. Lett. 76, 624 (1996).

[8] F. V. Hartemann et al., Phys. Rev. E 64, 016501 (2001).

[9] F. V. Hartemann, High-Field Electrodynamics (CRC Press, Boca Raton, FL, 2002), Chap. 10.

[10] Y. Y. Lau et al., Phys. Plasmas 10, 2155 (2003).

[11] W. J. Brown et al., Phys. Rev. ST Accel. Beams 7, 060702 (2004).

[12] W. J. Brown and F. V. Hartemann, Phys. Rev. ST Accel. Beams 7, 060703 (2004).

[13] F. V. Hartemann et al., Laser Part. Beams 22, 221 (2004).

[14] L. D. Landau and E. M. Lifshitz, Classical Theory of Fields (Butterworth-Heinemann, Oxford, U.K., 1975), 4 th ed.

[15] J. B. Rosenzweig, Fundamentals of Beam Physics (Oxford University Press, Oxford, U.K., 2003), Chap. 8.

[16] Table of Integrals, Series, and Products, edited by I. S. Gradshteyn and I. M. Ryzhik (Academic Press, San Diego, CA, 2000), 6th ed.

[17] S. Wolfram, The Mathematica Book (Wolfram Media, Champaign, IL and Cambridge University Press, Cambridge, U.K., 1999), 4th ed.

[18] C. A. Brau, Phys. Rev. ST Accel. Beams 7, 020701 (2004).

[19] G. A. Krafft, Phys. Rev. Lett. 92, 204802 (2004).

[20] J. Wu et al., Report No. SLAC-PUB-10430, 2004.

[21] http://www-ssrl.slac.stanford.edu/jhhome.html

[22] J. Welch and S. Anderson, Report No. SLAC-TN-02-001, 2002.

[23] C. P. J. Barty et al., Inst. Phys. Pub. IAEA Nucl. Fusion 44, S266 (2004). 